\title{
Goethe als Botaniker ${ }^{1}$
}

\section{Von OTto SchüePP}

Goethe soll als Botaniker gezeigt werden auf dem Hintergrund der Kenntnisse und Anschauungen seiner Zeit. Ich lasse ihn möglichst mit seinen eigenen Worten zu uns reden und zitiere nach «GoETHES naturwissenschaftliche Schriften in Auswahl» (Großherzog Wilhelm-ErnstAusgabe, Leipzig 1917). Aus der Literatur über Gozthe hebe ich hervor das Buch von Adolph Hansen: «Goethes Metamorphose der Pflanzen. Geschichte einer botanischen Hypothese» (Gießen 1907). Ergänzungen bringt Hansen : «Goethes Morphologie» (Gießen 1919). Ich empfehle das Studium von HANsen wegen der vielen Zitate aus älterer und neuerer Literatur, mahne aber zur Vorsicht gegenüber seinen Beurteilungen, die zwar immer zugunsten Gozthes ausfallen, aber doch keineswegs die wirkliche Bedeutung der Gedanken Goethes für uns deutlich machen. Den Versuch, «Goethes Erbe für die biologische Wissenschaft der Gegenwart fruchtbar zu machen» unternimmt Wilhelm Troll in "Gestalt und Urbild; Gesammelte Aufsätze zu Grundfragen der organischen Morphologie» (Leipzig 1941). So soll auch hier versucht werden, anhand von GoEthes Formulierungen unser gegenwärtiges naturwissenschaftliches und naturphilosophisches Forschen und Fragen zu klären.

GoEthe berichtet in Dichtung und Wahrheit: «Am 28. August 1749 mittags mit dem Glockenschlag $12 \mathrm{kam}$ ich in Frankfurt am Main auf die Welt. Die Konstellation war glücklich ... ) GoEthE legt Wert auf sein Horoskop; wir Naturwissenschaftler von heute achten nicht mehr auf die Stellung der Gestirne in der Geburtsstunde. Aber auch uns hat diese Stunde in eine bestimmte Zeit hineingestellt. Dies stellt uns unsere Aufgabe, gibt und begrenzt die Möglichkeiten für unsere Tätigkeit auf dem Felde der Wissenschaft.

Der alte GoEthe teilt 1831 die Geschichte seiner botanischen Studien mit: «In einer ansehnlichen Stadt geboren und erzogen, gewann ich meine erste Bildung in der Bemühung um alte und neuere Sprachen. Von dem hingegen, was eigentlich äußere Natur heißt, hatte ich keinen Begriff.» Als Staatsminister in Weimar hatte er sich mit Wäldern und Gärten zu beschäftigen. «Hiebei möchte man bemerken, daß der Gang meiner botanischen Studien einigermaßen der Geschichte der Botanik selbst ähnelte; denn ich war vom augenfälligsten Allgemeinsten auf das Nutzbare, Anwendbare, vom Bedarf zur Kenntnis gelangt.»

Zur wissenschaftlichen Botanik führte ihn der Apotheker Dr. BuchHolz: «Wohlhabend und lebenslustig, richtete dieser mit ruhmwürdiger Lernbegierde seine Tätigkeit auf Natur-

${ }^{1}$ Referat, gehalten an der Frühjahrsversammlung der schweizerischen botanischen Gesellschaft in Lugano, 14. März 194.9. 
wissenschaften. Als die naturforschende Welt sich eifrig beschäftigte, die verschiedenen Luftarten zu erkennen, versäumte er nicht, jederzeit das Neueste experimentierend vor Augen zu bringen. In seinen Gärten hatte er nicht die offizinellen Gewächse nur, sondern auch seltenere, neu bekannt gewordene Pflanzen für die Wissenschaften zu pflegen unternommen.» Der schon früh den Wissenschaften sich hingebende junge Regent ließ einen größeren botanischen Garten anlegen. «Unter solchen Umständen war auch ich genötigt, über botanische Dinge immer mehr und mehr Aufklärung zu suchen. Linnés Philosophie der Botanik war mein tägliches Studium.»

Auf der Reise nach Karlsbad läßt sich Goethe von dem Kräutersammler FriedRICH Gottlieb Dietrich begleiten, «der mit eifrigem Spürsinn alles Blühende zusammenbrachte, die Ausbeute womöglich an Ort und Stelle gleich in den Wagen hereinreichte und dabei nach Art eines Herolds die Linnéischen Bezeichnungen, Geschlecht und Art, mit froher Überzeugung ausrief, manchmal wohl mit falscher Betonung». Das fleißige Bemühen mit dem Sammeln und Einlegen von Pflanzen fand in der Badegesellschaft Freunde und Gegner. «Wir mußten öfters hören, die ganze Botanik sei nichts weiter als eine Nomenklatur und ein ganzes auf Zahlen gegründetes System; sie könne weder dem Verstand noch der Einbildungskraft genügen.»

Beim Eislauf, «auf der Schlittschuhbahn, damals dem Versammlungsort guter Gesellschaft», lernte Goethe den jungen August Karl Batsch kennen, und «besprach sich mit ihm über höhere Ansichten der Pflanzenkunde und über die verschiedenen Methoden, dieses Wissen zu behandeln, freimütig und anhaltend».

GoETHES eigene Gedanken reiften heran auf seiner italienischen Reise 1786-1788. Er gibt dazu «die ins Ganze greifende Bemerkung: daß alles, was uns von Jugend auf umgab, jedoch nur oberflächlich bekannt war und blieb, stets etwas Gemeines und Triviales für uns behält, das wir als gleichgültig neben uns bestehend ansehen, worüber zu denken wir gewissermaßen unfähig werden. Dagegen finden wir, daß neue Gegenstände in auffallender Mannigfaltigkeit, indem sie den Geist erregen, uns erfahren lassen, daß wir eines reinen Enthusiasmus fähig sind; sie deuten auf ein Höheres, welches zu erlangen uns wohl gegönnt sein dürfte. Dies ist der eigentliche Gewinn der Reisen.»

Im botanischen Garten zu Padua «zog eine Fächerpalme meine ganze Aufmerksamkeit auf sich; glücklicherweise standen die einfachen, lanzenförmigen ersten Blätter noch am Boden, die sukzessive Trennung derselben nahm zu, bis endlich das Fächerartige in vollkommener Ausbildung zu sehen war. Aus einer spathagleichen Scheide trat zuletzt ein Zweiglein mit Blüten hervor und erschien als ein sonderbares, mit dem vorhergehenden Wachstum in keinem Verhältnis stehendes Erzeugnis, fremdartig und überraschend».

In Sizilien und Rom reifen die Gedanken über die Metamorphose heran. Nach der Rückkehr wird im Dezember 1789 der erste Entwurf fertig. 1790 erscheint J.W. von Goethe, Herzoglich Sachsen-Weimarschen Geheimrates Versuch, die Metamorphose der Pflanzen zu erklären - (Gotha, bei KarL Wilhelm EtTinger). 1817 berichtet Goethe: «Mit meinem neuen Hefte wohl zufrieden, schmeichelte ich mir, auch im wissenschaftlichen Felde schriftstellerisch eine glückliche Laufbahn zu eröffnen.» Aber er wurde mit dem Heft von seinem bisherigen Verleger Göschen abgewiesen. «Das Publikum stutzte; denn nach seinem Wunsche, sich gut und gleichförmig bedient zu sehen, verlangt es an jeden, daß er in seinem Fache bleibe.» Über Rousseaus botanische Schriften urteilt Goethe 1831, «daß es die Erfahrung gäbe, daß Dilettanten zum Vorteil der Wissenschaft vieles 
beitragen. Männer vom Fach müssen sich um Vollständigkeit bemühen, dem Liebhaber dagegen ist es darum zu tun, durch das Einzelne durchzukommen und einen Hochpunkt zu erreichen, von woher ihm eine Übersicht wo nicht des Ganzen, doch des Meisten gelingen könnte.»

Was dachte man über die Metamorphose vor Goethe? Linné sagt in seinen «Amoenitates academicae IV 368» (übersetzt bei HANSEN, S. 189) in Anknüpfung an Andrea Caesalpino 1583 und an Swammerdams «Bibel der Natur» 1737: «Die Pflanzen sind der gleichen Umwandlung wie die Tiere und einer nicht minder bewunderungswürdigen Metamorphose unterworfen. Die Metamorphose der Insekten besteht in der Abwerfung einer oder mehrerer Häute, indem sie darauf in ihrer eigentlichen und vollkommenen Form nackt hervorkommen ... Die Rinde der Pflanze verhält sich so wie die Hülle der Larve ... Die Blüten treten aus der geöffneten Rinde hervor... Solange die Pflanze innerhalb der Rinde liegt, nur mit Blättern bekleidet, ist sie uns genau so unbekannt und dunkel wie ein Schmetterling, solange er Larve ist ... Die Insekten leben lange als Larve, aber nachdem sie verwandelt sind, beeilen sie sich, ihr Geschlecht fortzupflanzen. In gleicher Weise verhalten sich auch die Pflanzen, welche fast ihr ganzes Leben lang die Erde mit grünenden Blättern bedecken und, nur kurze Zeit, aus ihrer Larve herauskommend blühen und fruchten.» Auch Goethe findet: (1820) «Die Verwandlung der Insekten an und für sich genau zu betrachten und mit der Pflanzenverwandlung zu vergleichen, wird ein sehr angenehmes Geschäft sein.»

Wenn LinNé im besondern den Kelch aus der Rinde, die Krone aus dem Bast, Staubgefäße, Stempel und Fruchtgehäuse aus dem «Eingeweide», Holz und Mark des Zweiges hervorgehen läßt, so weiß GoEthe (Metamorphose § 111): «Die zweite Rinde (mit dem Cambium) ist es, welche alle Kraft des Lebens und Wachstums enthält.»

Ein anderer Gedankengang LiNNÉs knüpft an die Verzweigung der Bäume an. «Die Knospen bestehen aus Blattanfängen mit ihren Knöspchen, und diese Knöspchen wieder aus Blättchen mit ihren Knöspchen . . .» Bei der Blütenbildung sollen sechs Jahrgänge von Blättern vorzeitig und umgewandelt als Brakteen, Kelch, Krone, Staubgefäße, Stempel und Fruchtgehäuse hervortreten. Goethe lehnt diese Lehre von der «Prolepsis» ab (Met. 109). «Nach LiNNÉs Lehre müßte man annehmen, daß jede einjährige Pflanze eigentlich von der Natur bestimmt gewesen sei, sechs Jahre zu wachsen, und diese längere Frist in dem Blüten- und Frucht- 
stande einmal antizipiere und sodann verwelke.» GoETHE hat den bessern Ausgangspunkt gewählt (Met. 110): «Wir sind dagegen zuerst dem Wachstum der einjährigen Pflanze gefolgt; nun läßt sich die Anwendung auf die dauernden Gewächse leicht machen, da eine aufbrechende Knospe des ältesten Baumes als eine einjährige Pflanze anzusehen ist.»

Goethe beginnt seine Einleitung (§ 1-9) mit dem Satz: «Ein jeder, der das Wachstum der Pflanzen nur einigermaßen beobachtet, wird leicht bemerken ...» Die Wahrnehmung der Metamorphose setzt aufmerksame Beobachtung voraus, verlangt aber keine technischen Hilfsmittel. Man bemerkt, «daß gewisse äußere Teile der Pflanze sich manchmal verwandeln und in die Gestalt der nächstliegenden Teile bald ganz, bald mehr oder weniger übergehen.»

Das beobachtende Auge des Morphologen springt oder gleitet von einem Teil zum nächstliegenden hinüber, wandert am Stengel aufwärts von Blatt zu Blatt, vom Kelch zur Krone. Von einer gesehenen Gestalt bleibt im Auge oder im Gedächtnis des Beobachters ein Nachbild, das mit dem neu ins Auge gefaßten Bild zur Deckung gebracht wird. Während das äußere Auge über eine Reihe selbständiger Gestalten hinweggleitet, sieht das innere Auge die Metamorphose einer Gestalt. Dieser Vorgang spielt sich rasch und unbemerkt ab bei jeder Kinovorführung.

Goethe definiert im $\S 4:$ :Die geheime Verwandtschaft der verschiedenen äußeren Pflanzenteile, als der Blätter, des Kelches, der Krone, der Staubfäden, welche sich nacheinander und gleichsam auseinander entwickeln, ist von den Forschern im allgemeinen längst erkannt, ja auch besonders bearbeitet worden, und man hat die Wirkung, wodurch ein und dasselbe Organ sich uns mannigfaltig verändert sehen läßt, die Metamorphose der Pflanzen genannt.» Wir beachten das Wörtchen «gleichsam»: Was sich im Überblick als eine ununterbrochen fortschreitende Verwandlung der Gestalt darbietet, ist die Folge einer «geheimen», verborgenen, inneren Umwandlung, die «nacheinander» verwandte Gestalten hervorbringt. Hinter der Erscheinung steht eine «Wirkung» und auch diese bezeichnet Gozthe als Metamorphose.

Die Metamorphose ist «regelmäßig», «unregelmäßig» oder «zufällig». Wir gehen mit GoETHE rasch an der «zufälligen», «von außen», besonders durch Insekten bewirkten Metamorphose mit ihren «monströsen Auswüchsen» vorbei (§ 8). Die «unregelmäßige» oder «rückschreitende» Metamorphose ist methodisch wichtig $(\S 7)$, weil wir durch die Erfahrungen, welche wir an ihr zu machen die Gelegenheit haben, dasjenige enthüllen können, was 
uns die regelmäßige Metamorphose verheimlicht. Als Beispiel nennt Gozтне die Veränderung der einfachen Blume in eine gefüllte, «wenn sich anstatt der Staubfäden und Staubbeutel Blumenblätter entwickeln, die entweder an Gestalt und Farbe vollkommen den übrigen Blättern der Krone gleich sind oder noch sichtbare Zeichen ihres Ursprungs an sich tragen». Während hier die Natur «um eine oder einige Stufen rückwärts tritt», eilt sie in der regelmäßigen Metamorphose vorwärts, auf ihren großen Zweck hin.

Die Metamorphose ist eine gerichtete Umwandlung; sie schreitet normalerweise vorwärts und ist nur in beschränktem Umfange umkehrbar. Sie ist nicht bloß ein Ablauf, der auf einen Ausgleich, einen Gleichgewichtszustand hinzielt, sondern ein «Hineilen zu einem großen Zwecke» (§ 6). «Die regelmäßig fortschreitende Metamorphose macht sich von den ersten Samenblättern bis zur letzten Ausbildung der Frucht immer stufenweise wirksam bemerkbar. Sie steigt durch Umwandlung einer Gestalt in die andere gleichsam auf einer geistigen Leiter zu jenem Gipfel der Natur, der Fortpflanzung durch zwei Geschlechter hinauf. Diese ist es, welche zu erklären ich gegenwärtigen Versuch unternehme.»

Welcher Art ist die Erklärung, die uns Goethe verspricht? Nach was für Gesetzen bringt die Natur «einen Teil durch den andern hervor?» (§3) Wird die Erklärung wissenschaftlich sein, oder versteigt sich GoEthE mit seiner Rede von der «geistigen Leiter» in abstrakte Naturphilosophie? Goethe mußte bald hören, daß seine Arbeit nicht als wissenschaftlich anerkannt wurde (1817). Einer seiner römischen Kunstfreunde kam ihm zu Hilfe mit der Deutung: «Er will die Künstler lehren, wie sprossende und rankende Blumenverzierungen zu erfinden sind.» «Nirgends wollte man zugeben, daß Wissenschaft und Poesie vereinbar sind. Man vergaß, daß Wissenschaft sich aus Poesie entwickelt habe; man bedachte nicht, daß nach einem Umschwung von Zeiten beide sich wieder freundlich, zu beiderseitigem Vorteil, auf höherer Stelle, gar wohl wieder begegnen könnten.» Freundinnen sucht er durch eine Elegie zur Teilnahme an seinen wissenschaftlichen Bestrebungen zu locken:

«Alle Gestalten sind ähnlich, und keine gleichet der andern, Und so deutet das Chor auf ein geheimes Gesetz Auf ein heiliges Rätsel. O, könnt' ich dir, liebliche Freundin, Überliefern sogleich glücklich das lösende Wort!»

Kunstvoll wird der Gang der Metamorphose in Versen geschildert bis zu ihrem Abschluß in der Blüte: 


\title{
«Traulich stehen sie nun, die holden Paare beisammen, Zahlreich ordnen sie sich um den geweihten Altar.»
}

Was an der Pflanze geschaut wird, findet seine Anwendung auf das menschliche Leben, auf Bekanntschaft, Freundschaft, Liebe:

\author{
«Die heilige Liebe
}

Strebt zu der höchsten Frucht gleicher Gesinnungen auf, Gleicher Ansicht der Dinge, damit in harmonischem Anschaun Sich verbinde das Paar, finde die höhere Welt.»

Man ist verwundert, in einer Schrift Goethes über «Verstäubung, Verdunstung, Vertropfung» aus dem Jahre 1820 ganz andere Töne zu hören. Auf den Wegen des großherzoglichen botanischen Gartens tat ihm Professor Schelver «vertraulichste Eröffnung, daß er an der Lehre, welche den Pflanzen wie den Tieren zwei Geschlechter zuschreibt, längst gezweifelt habe und nun von ihrer Unhaltbarkeit völlig überzeugt sei». Auch GoETHE ließ sich überzeugen und stimmt der neuen Verstäubungslehre zu. "Im ruhigen Gang der Metamorphose bleibt alles Stoffartige, Geringere, Gemeinere nach und nach zurück.» Die Ausschüttung des Blütenstaubes wird zu einer Befreiung vom lästigen Stoff. Sie ist vergleichbar dem «Geruch des Berberitzenstrauches, der nahe gelegene Weizenfelder unfruchtbar machen kann». «Brand im Korn und Mais» und die «zerstörende Verstäubung der Fliegen im Herbst»" gehören in den gleichen Kreis von Erscheinungen. (Die Pilzkunde ist noch weit im Rückstand.) «Diese neue Verstäubungslehre wäre nun beim Vortrage gegen junge Personen und Frauen höchst schicklich: denn der persönlich Lehrende war bisher durchaus in großer Verlegenheit. Die ewigen Hochzeiten, die man nicht los wird, wobei die Monogamie, auf welche Sitte, Gesetz und Religion gegründet sind, ganz in eine vage Lüsternheit sich auflöst, bleiben dem reinen Menschensinne völlig unerträglich.»

Der jüngere GoEthe, der in der geschlechtlichen Differenzierung den Höhepunkt der Metamorphose sah, hat gegenüber Schelver und dem älteren GoETHE Recht behalten. Was das Wesen des männlichen oder weiblichen Zustandes im Protoplasma sei, können wir auch heute nicht sagen. Geschlechtliche Differenzierung ist ein Urphänomen in der Biologie, wie der Gegensatz positiver und negativer Elektrizität ein Urphänomen in der Physik ist. Die Sexualität wird am deutlichsten sichtbar an den Fortpflanzungsorganen, den Bildungsstätten der Gameten und an den Gameten selbst. Die Sexualität wird nicht erklärt durch die Vorteile der Amphimixis für die Ausbreitung günstiger Mutationen. Die «sekundären» Geschlechtsmerkmale werden nicht erklärt durch ihre Mitwirkung bei der Herbeiführung der Befruchtung. Sexualität wird von uns Menschen nicht nur in der Natur beobachtet, sondern auch als Trieb erlebt. Sie gehört zu den Grundkräften unseres Seelenlebens und wirkt sich aus in den verschiedensten Lebensgebieten.

Wenn GоEтнE erwartete, «daß nach einem Umschwung der Zeiten» sich Wissenschaft und Poesie wieder «freundlich begegnen» möchten, so mag das zuerst auf dem Gebiete der Psychologie erfolgen.

Mit Linné und Goethe werden wir auch heute sagen müssen, daß die Metamorphose aus einem indifferenten Jugendzustande nach der Ent- 
faltung männlicher und weiblicher Sexualität hinstrebt und in den Fortpflanzungsorganen ihren sichtbaren Höhepunkt erreicht. Auch wir sind geneigt, in der Metamorphose einen autonomen Vorgang zu sehen, dessen wesentliche Ursachen in der Pflanze selbst liegen. Wir werden aber auch, wieder im Anschluß an Gozthe, nach den Beziehungen der Metamorphose zur Umwelt, nach den Abhängigkeiten der Metamorphose vom Stoff- und Energiewechsel der Pflanze fragen.

In elf Abschnitten über die Samenblätter, die Ausbildung der Stengelblätter von Knoten zu Knoten, den Übergang zum Blütenstand, die Bildung des Kelches und der Krone, der Staubwerkzeuge, der Nektarien, des Griffels, der Früchte und der unmittelbaren Hüllen der Samen beschreibt GoEтHE die regelmäßig fortschreitende Metamorphose, meist in allgemeinen Zügen, gelegentlich unter Nennung einzelner Pflanzenarten. «Die vornehmste Ursache der mannigfaltigen Blattgestalten» findet er «im Verhältnis der Rippen gegeneinander». Je nach der Ausbildung von Mittelrippe und Seitenrippen «erscheinen die Blätter nurmehr eingekerbt, tief eingeschnitten, aus mehreren Blättchen zusammengesetzt». In der Beschreibung wird der Begriff der «Anastomose» verwendet für die Gefäße, die sich mit ihren Enden aufsuchen, auch für die «in ihrem zarten Zustande anastomosierenden Kelchblätter, welche die glockenförmigen oder sogenannten einblättrigen Kelche» darstellen. Vielfach wird hingewiesen auf den Wechsel von Ausdehnung und Zusammenziehung (§ 115). «Dasselbe Organ, welches am Stengel sich ausgedehnt und eine höchst mannigfache Gestalt angenommen hat, zieht sich nun im Kelch zusammen, dehnt sich im Blumenblatt wieder aus, zieht sich in den Geschlechtsorganen zusammen, um sich als Frucht zum letztenmal auszudehnen.» Dazu kommt die «Versammlung» verschiedener Organe um ein Zentrum, den Blütenboden.

Goethe sagt, daß er die Metamorphose bei der Beobachtung des Wachstums gefunden habe (§ 1). Er macht aber kaum eigentliche Wachstumsbeobachtungen, sondern vergleicht die nacheinander aus derselben Knospe hervorgetretenen ausgewachsenen Teile. Vor ihm hat 1759 KaSPar FriEDERICH WOLFF in seiner «Theoria generationis» die Vegetationspunkte und die Bildung der Blattanlagen als Anhängsel des Stammes beschrieben. Gozthe ist später, nach der Abfassung seiner Schrift über die Metamorphose, mit WoLfFs Arbeiten bekannt geworden und würdigt ihn 1817 als einen "trefflichen Vorarbeiter», druckt auch einen längeren Abschnitt aus einer Arbeit Wolffs ab. Auch WolfF schließt aus vergleichender Betrachtung, «daß alle Teile der Pflanze, den Stengel ausgenommen, auf die 
Form des Blattes zurückgeführt werden können und nichts als Modifikationen desselben sind». WolfF fordert: «Zuerst muß durch Beobachtungen ausgemittelt werden, auf welche Weise die gewöhnlichen Blätter sich bilden. Nachher erst ist die Frage zu stellen, welche Umstände und Bedingungen in den oberen Teilen der Pflanze die allgemeine Vegetationsweise so modifizieren, daß an Stelle gewöhnlicher Blätter diese eigentümlich gebildeten zum Auftritt kommen.» WolfF deutet diese Modifikation allzu einfach als «allmähliche Abnahme der Vegetationskraft.»

Goethe weigert sich, seinem Vorgänger in der Methode zu folgen. Denn dieser «setzt als Grundmaxime aller seiner Forschungen, daß man nichts annehmen, zugeben und behaupten könne, als was man mit Augen gesehen und andern jederzeit wieder vorzuzeigen imstande sei. Deshalb ist er immer bemüht, auf die Anfänge der Lebensbildung durch mikroskopische Untersuchungen zu dringen, und so die organischen Embryonen von ihrer frühesten Erscheinung bis zur Ausbildung zu verfolgen. Wie vortrefflich diese Methode auch sei, so dachte der treffliche Mann doch nicht, daß es ein Unterschied sei zwischen Sehen und Sehen, daß die Geistesaugen mit den Augen des Leibes in stetem lebendigen Bunde zu wirken haben, weil man sonst in Gefahr gerät zu sehen und doch vorbeizusehen.»

Die Frage an uns lautet, ob nicht die starke Konzentration auf die entwicklungsgeschichtliche Forschung, wie sie CARL Nägeli vor hundert Jahren einleitete, und wie sie heute unter Ausnutzung von Mikrotomserien erneut gepflegt wird, die Aufmerksamkeit ablenke von den Beziehungen, welche die vergleichende Betrachtung erwachsener Formen nachweist. Mit wachsender Vergrößerung und abnehmender Schnittdicke verengert sich das Gesichtsfeld des Mikroskopikers, und es bedarf besonderer Bemühungen, um die Erscheinungen aller Größenordnungen von der Meristemzelle bis zum Baum gleichzeitig vor Augen zu haben.

Die Konkurrenz zwischen der entwicklungsgeschichtlichen Forschung, die allen Veränderungen am Einzelobjekt von Nahem nachspürt, und zwischen dem vergleichenden Überblick, der aus vielen Einzelformen den «Typus» mit seinen Wandlungen erfaßt, geht seit WolfF und Goethe durch die botanische Forschung. Schleiden nimmt 1843 die Forderungen WolfFs auf und lehnt Goethes Metamorphosenlehre als Irrweg ab: «Die unglückselige Saat, die GoEthe gesäet, wucherte mit trauriger Schnelligkeit auf und nächst dem Schellingianismus verdanken wir es ihm, daß Phantasiespiele in der Botanik an die Stelle ernster und scharfer Wissenschaftlichkeit getreten sind.» 
Troll betont mit Celakovsky (1876) und Braun (1875), gegen GréGorre (1931) und Foster (1928) immer wieder, daß die Entwicklungsgeschichte «nur das Material liefert, das erst in komparativer Weise für morphologische Schlüsse verarbeitet werden muß». GoEBEL glaubt, daß die Anlagen gewöhnlicher Laubblätter durch Veränderung der Wachstumsbedingungen, welche sie in frühem Zustande treffen, umgebildet werden, z. B. zu Knospenschuppen; er wiederholt damit die Gedanken von WoLfF. Nachuntersuchung (ScHÜEPP 1911) zeigt aber, daß die Umbildung schon die ersten sichtbaren Anlagestadien erfaßt hat, daß sie mit der Entstehung erfolgt, «kongenital» ist. Erst recht erweist sich die Umbildung vom vegetativen Sproß zum Blütenstand (GrÉGoIre 1938, SснÜEPP 1942) als eine kongenitale Metamorphose des ganzen Entwicklungsganges. Es sind nicht nur Formen, sondern Wachstumsordnungen, die wir zu vergleichen und soweit als möglich zu erklären haben.

Wenn wir mit Goethe (§ 4) die Metamorphose als eine «Wirkung» betrachten wollen, so haben wir nicht nur auf die Formen und den Formwechsel zu achten, sondern auch auf das Material, aus welchem die Formen gebaut werden, und auf die physiologischen Prozesse, mit welchen die Formbildung verknüpft ist. Auch der Morphologe darf nicht vergessen, daß die Pflanze aus Nährstoffen und mit Hilfe von Energie und Reizwirkungen der Umwelt ihre Formen baut. Wir lesen bei Goethe (Met. $\S 12)$ : «Die Kotyledonen erscheinen oft unförmlich, mit einer rohen Materie gleichsam ausgestopft» $(\S 24):$ «Wie nun die Blätter hauptsächlich ihre erste Nahrung den mehr oder weniger modifizierten wässerichten Teilen zu verdanken haben, welche sie dem Stamme entziehen, so sind sie ihre größere Ausbildung dem Lichte und der Luft schuldig» (§ 26): «Man hat sich durch Erfahrung unterrichtet, da $\beta$ die Blätter verschiedene Luftarten einsaugen und sie mit den in ihrem Innern enthaltenen Feuchtigkeiten verbinden; auch bleibt wohl kein Zweifel übrig, daß sie diese feineren Säfte wieder in den Stengel zurückbringen und die Ausbildung der in ihrer Nähe liegenden Augen vorzüglich befördern» (§ 27): «Ein oberer Knoten, indem er aus dem vorhergehenden entsteht und die Säfte unmittelbar durch ihn empfängt, wird solche feiner und filtrierter erhalten, auch von der inzwischen geschehenen Einwirkung der Blätter genießen, sich selbst feiner ausbilden und seinen Blättern und Augen feinere Säfte zubringen.»

Erfahrungen über Ernährung und Blütenbildung werden in die Theorie mit eingebaut $(\S 30)$. «Solange (durch die Tätigkeit der Stammblätter) noch rohere Säfte abzuführen sind, solange müssen sich die möglichen 
Organe der Pflanze zu Werkzeugen dieses Bedürfnisses ausbilden. Entzieht man der Pflanze die Nahrung, so erleichtert und verkürzt man dagegen jene Wirkung der Natur; die Organe der Knoten werden verfeinert, die Wirkung der unverfälschten Säfte reiner und kräftiger, die Umwandlung der Teile wird möglich und geschieht unaufhaltsam.»

Während Goethe so um 1790 über Ernährung und Metamorphose spekulierte, schufen Ingen-Houss, Senebier und Saussure 1796 bis 1804 die heute noch gültige Lehre von der Ernährung der Pflanzen aus Kohlendioxyd und Wasser mit Hilfe der vom Chlorophyll absorbierten Lichtenergie. Wir finden nicht, daß sich GoEthe je mit dieser Lehre auseinandergesetzt habe. Auch seine Kenntnisse von der Pflanzenzelle bleiben spärlich und ohne rechten Zusammenhang. Wir erfahren von einem «zelligen Gewebe», welches das Mark ausfüllt (§27). Eine merkwürdige Bedeutung wird den Spiralgefäßen zugeschrieben $(\S 60,61)$. «Daß die Geschlechtsteile der Pflanze durch die Spiralgefäße wie die übrigen Teile hervorgebracht werden, ist durch mikroskopische Beobachtungen außer allen Zweifel gesetzt. Wir nehmen daraus ein Argument für die innere Identität der verschiedenen Pflanzenteile, welche uns bisher in so mannigfachen Gestalten erschienen sind.» Ihre Wirkung wird zunächst recht mechanistisch vorgestellt. «Sie erscheinen uns wirklich als elastische Federn, welche durch ihre Spannkraft die umschließenden Gewebe dehnen und zum Wachsen bringen.» In dem späteren Aufsatz über die Spiraltendenz aus dem Jahr 1831 finden wir den Spiralgefäßen die Bedeutung zugeschrieben, welche für uns die Zellen erlangt haben. "Wir betrachten sie hier als die kleinsten Teile, welche dem Ganzen, dem sie angehören, vollkommen gleich sind und als Homoiomerien anzusehen, ihm ihre Eigenheiten mitteilen und von demselben wieder Eigenschaft und Bestimmung erhalten.»

Im Jahr 1949 reden wir von Protoplasma, von Chromosomen und Genen. Statt von «roheren und feineren Säften» reden wir vom Zellsaft mit anorganischen Salzen, mit Zuckern, Aminosäuren, auch von besonderen Säften wie dem Auxin, oder hypothetisch von Blühhormonen. Wie verhält es sich mit einem Versuch, nach Goethes Vorbild die Metamorphose zu erklären, wenn wir an gegebener Stelle unsere modernen anatomischen und biochemischen Kenntnisse einsetzen?

GoEthe faßt seine Gedanken im Rückblick (§ 84) folgendermaßen zusammen: «Und so wären wir der Natur auf ihren Schritten so bedachtsam als möglich gefolgt; wir hätten die äußere Gestalt der Pflanze in allen ihren Umwandlungen von ihrer Entwicklung aus dem Samenkorn bis zur neuen Bildung 
desselben begleitet und, ohne Anmaßung, die ersten Triebfedern der Naturwirkung entdecken zu wollen, auf Äußerung der Kräfte, durch welche die Pflanze ein und eben dasselbe Organ nach und nach umbildet, unsere Aufmerksamkeit gerichtet.» Handelndes Subjekt ist nach diesem charakteristischen Satze von Goethe «die Natur» oder «die Pflanze». Die Natur erreicht ihre Ziele durch Einsatz von Kräften und Stoffen als Mitteln. Unserer Zeit ist das eine fremde Ausdrucksweise geworden. SAcHs spricht es 1882 aus, daß «Stoff und Form» der Pflanzenorgane so zusammengehören, daß die Form mit Notwendigkeit aus den Eigenschaften des Stoffes hervorgeht. Nach SaGhS oder Klebs müßten die «ersten Triebfedern» für den Vorgang der Metamorphose in den allgemeinen Eigenschaften des Stoffes und in den Grundgesetzen der Energetik gefunden werden. Für den Experimentator ist die jeweils angewandte Kombination von Nährstoffen, Temperatur und Licht die Ursache; die beobachtete Wachstumsform Folge. Der Morphologe kann sich aber auch heute mit einer solchen Formulierung nicht begnügen. Ihm bleibt wesentliche Ursache der Gestalt ein autonomes Bildungsbestreben (nicht bloß eine ererbte Reaktionsfähigkeit). Aber der Bildungstrieb, der «nisus formativus» von Blumenbach und Goethe (1820) braucht Mittel zur Verwirklichung und er ist in seiner Tätigkeit abhängig von den Mitteln. Es sind dieselben Stoffe, Kräfte, Bedingungskomplexe, welche in der Betrachtungsweise des Experimentators als eigentliche «Ursachen» der Formbildung und Umbildung gewertet werden, und welche dem Morphologen als die «Mittel» zur Verwirklichung der Formen erscheinen. «Mittel» sind nicht letzte Ursachen, sondern sie sind eingeordnet in eine Kette von Vorgängen, die früher beginnt und weiter reicht als unsere unmittelbare Kenntnis von Stoffwechsel und Energiewechsel.

Der Hammer mit seiner kinetischen Energie und der Meißel mit seiner Härte und Schärfe sind unmittelbare Ursachen dafür, wie Stücke von einem Marmorblock absplittern. In der Hand des Künstlers sind Hammer und Meißel die Mittel, mit denen er eine zuvor als Idee erschaute Gestalt aus der Materie herausarbeitet. Was der Künstler greifbar und sichtbar darstellen kann, ist auch abhängig von den Mitteln, die ihm zur Verfügung stehen. Wir müssen den Einfluß der Mittel auf die erstrebte Form bei den organischen Formen in der Natur hoch einschätzen; die Mittel sind auch Mitursachen der Form.

Was hat Goethes Metamorphosenlehre Neues gebracht, und wie wirkte sich dieses in der Wissenschaft aus? 
Während GoEthE auf seiner italienischen Reise der Begriff der «sinnlichen Form einer übersinnlichen Urpflanze vorschwebte», schrieb ВАтscH seinen «Versuch einer Anleitung zur Kenntnis und Geschichte der Pflanzen für akademische Vorlesungen entworfen» (Halle 1787). In den Paragraphen 292 bis 297 heißt es unter anderem: «Aber nicht allein die Säfte, auch die Gestalt des Gewächses wird während dem fortgehenden Lauf der Nahrung verändert ... Durch die ganze Pflanze sehen wir diese blattähnlichen Körper, oft im sichtlichen Übergang und der deutlichsten Verwandtschaft, in Gestalt, Wesen und Farbe ... Die allzu häufige Nahrung macht, daß die Triebe rückwärts gehen, und sich wieder von ihrer vorigen Fortschreitung entfernen ... N Nachdem GoETHE sein Manuskript der Metamorphose BATSCH zur Einsicht vorgelegt hatte, schreibt ihm dieser am 19. Januar 1790 (siehe bei Hansen, Met. S. 364): als «Ew. Hochwohlgebornen Excellenz untertänigster Diener» ..., «daß es das freiwilligste Bekenntnis sei, wenn ich sage, daß jene edlen Analogien, mich, im ganzen genommen überrascht und hingerissen haben. Sie sind weiter auf diesem Wege gegangen, als ich versuchte; schon weiß ich es nicht mehr, ob wir uns vor fünf Jahren auf ihm begegneten, oder ob ich es ganz Ihrem Winke schuldig bin, auf ihn gekommen zu sein; eines wie das andere ist für mich ehrenvolle und teure Erinnerung.» In der «Botanik für Frauenzimmer und Pflanzenliebhaber» (Weimar 1795) wiederholt BAtsch im $\S 67$ kurz die Metamorphosenlehre. Goethe erwähnt er nicht, sondern preist auf der Schlußseite Rousseau, «den unvergeßlichen Bürger von Genf», als Vorgänger. War BATSCH doch unbefriedigt von dem kurzen Zitat über die «schon einzeln gemachten, auch gesammelten und gereiheten Beobachtungen», für welche GoETHE auf BATsch verweist (Met. § 112)? GoETHE spricht sich 1820 über Prioritätsfragen aus: «Manchmal ziehen gewisse Gedanken schon in der Luft herum, so daß mehrere sie erfassen können.» «Wer es mit sich herumträgt, muß davon sprechen, er sucht andern seine Überzeugungen aufzudringen, er wird nicht anerkannt. Endlich ergreift es ein Fähiger und bringt es mehr oder weniger als sein Eigenes vor.»

Im Vergleich zu Batsch war sicher GoEthe der Fähigere, dem es gegeben wurde, den Gedanken der Metamorphose in der Geschichte der Botanik zur Geltung zu bringen. Am Beginn dieser Geschichte steht im Jahr 1794 ein «Glückliches Ereignis», das GoEthe 1817 erzählt: «Aus Italien zurückgekehrt, fand ich neuere und ältere Dichterwerke in großem Ansehen, von ausgebreiteter Wirkung, leider solche, die mich äußerst anwiderten, ich nenne nur Heinses Ardinghello und Schillers Räuber ... Schiller zog nach Jena ... Zu gleicher Zeit hatte BATScH durch unglaubliche Regsamkeit eine naturforschende Gesellschaft in Tätigkeit gesetzt. Ihren periodischen Sitzungen wohnte ich gewöhnlich bei; einstmals fand ich ScHillern daselbst, wir gingen zufällig beide zugleich heraus, ein $\mathrm{Ge}$ spräch knüpfte sich an.» Schiller kritisierte die zerstückelte Art, die Natur zu behandeln. GoEthe betonte die Möglichkeit, «die Natur wirkend und lebendig, aus dem Ganzen in die Teile strebend», darzustellen. Er trat bei Schiller ein. «Da trug ich die Metamorphose der Pflanzen lebhaft vor 
und ließ mit manchem charakteristischen Federstrich eine symbolische Pflanze vor seinen Augen entstehen. Als ich aber geendet hatte, schüttelte er den Kopf und sagte: Das ist keine Erfahrung, das ist eine Idee. Ich stutzte, verdrießlich einigermaßen; ich nahm mich aber zusammen und versetzte: Das kann mir sehr lieb sein, daß ich Ideen habe, ohne es zu wissen, und sie sogar mit Augen sehe. Schiller erwiderte darauf als gebildeter Kantianer, und als aus meinem hartnäckigen Realismus mancher Anlaß zu lebhaftem Widerspruch entstand, so ward viel gekämpft und dann Stillstand gemacht; keiner von beiden konnte sich für den Sieger halten, beide hielten sich für unüberwindlich.»

Wir haben vielleicht heute von der theoretischen Physik her gelernt, daß es sich zwischen Schiller und Goethe um ein Gespräch handelt, das gar nicht mit dem Siege einer Auffassung abgeschlossen werden kann, sondern fortdauern muß. Wir achten noch auf einige weitere Beiträge GoEthES zu diesem Gespräch. 1817 schreibt er: «Wir glauben zu einer Kenntnis des Wesens und Wirkens der Naturgegenstände am besten durch Trennung der Teile gelangen zu können; wie denn auch wirklich dieser Weg uns sehr weit zu führen geeignet ist (Chemie, Anatomie). Aber diese trennenden Bemühungen, immer und immer fortgesetzt, bringen auch manchen Nachteil hervor. Das Lebendige ist zwar in Elemente zerlegt, aber man kann es aus diesen nicht wieder zusammenstellen und beleben. Es hat sich daher auch in dem wissenschaftlichen Menschen zu allen Zeiten ein Trieb hervorgetan, die lebendigen Bildungen als solche zu erkennen, ihre äußern sichtbaren, greifbaren Teile im Zusammenhange zu erfassen, sie als $A n$ deutungen eines Innern aufzunehmen und so das Ganze in der Anschauung gewissermaßen zu beherrschen.» Darum wird versucht, eine Lehre auszubilden, welche wir Morphologie nennen möchten (siehe Troll, Anm.147). Der Deutsche hat für den Komplex des Daseins eines wirklichen Wesens das Wort Gestalt. Wir finden, daß nirgend ein Bestehendes, nirgend ein Ruhendes, ein Abgeschlossenes vorkommt, sondern daß vielmehr alles in einer steten Bewegung schwanke. Daher unsere Sprache das Wort Bildung sowohl vom Hervorgebrachten als vom Hervorgebrachtwerdenden gehörig genug zu brauchen pflegt.»

«Jedes Lebendige ist kein Einzelnes, sondern eine Mehrheit; selbst insofern es uns als Individuum erscheint, bleibt es doch eine Versammlung von lebendigen selbständigen Wesen, die der Idee, der Anlage nach gleich sind, in der Erscheinung aber gleich oder ähnlich, ungleich oder unähnlich werden können.» Zwischen Idee und Erscheinung schaltet GoEthe den 
Begriff der Anlage ein. Er meint damit etwas, das von der unsichtbaren, nicht greifbaren Idee vermittelnd hinüberführt zum sichtbaren, greifbaren, körperlichen Organ. Anlage ist das Glied im Stande des Hervorgebrachtwerdens. Der Begriff der Anlage hat in der Wissenschaft wechselnden Inhalt und Umfang. Beim Gen denken wir an Teilchen im Chromosom, die wir zwar nicht chemisch definieren können, die wir aber doch auf ihrem Wege durch Mitosen und Meiose und Befruchtung verfolgen und an ihrer Wirkung auf die Ausbildung bestimmter Merkmale erkennen können. Oder. wir denken bei einer Blattanlage an einen Zellhügel aus ein paar hundert gleichartigen Meristemzellen. Wir reden von Anlagen, aber kaum mehr von Ideen. Troll meint (l. c. S. 71): «Ideen haben im mechanistischen Weltbild, wie es sich namentlich seit dem Auftreten KaNTS entwickelt und unter dem Einfluß der exakten Wissenschaften ausgestaltet hat, keinen Platz, außer als ,regulative Prinzipien', die den Verstand in der Betrachtung der Natur nach einem Prinzip der Vollständigkeit zu leiten vermögen.»

Für Goethe aber bedeutet die Idee mehr als eine dem klaren Begriff vorauseilende Funktion unseres Denkens; ihm ist die Idee etwas in der Natur Wirkendes. «Daß nun das, was der Idee nach gleich ist, in der Erfahrung entweder als gleich oder als ähnlich, ja sogar als völlig ungleich und unähnlich erscheinen kann, darin besteht eigentlich das bewegliche Leben der Natur.»

Wir werden bei der Beschreibung der Entwicklungsvorgänge immer zwischen den Begriffen der Präformation und der Epigenesis hin und her unsern Weg suchen müssen. GoEthe sagt von seiner Zeit 1817: «Die Einschachtelungslehre, der Begriff von Präformation, von sukzessiver Entwicklung des von Adams Zeiten her schon Vorhandenen hatten sich selbst der besten Köpfe im allgemeinen bemächtigt.» Er muß selber zugeben (1820): «Und wenn wir keine Präformation denken wollen, so kommen wir auf eine Prädelineation, Prädetermination, auf ein Prästabilieren, und wie das alles heißen mag, was vorausgehen müßte, bis wir etwas gewahr werden können. Der Begriff der Epigenesis aber führt auf den Begriff der Kraft, der zunächst etwas nur Physisches, sogar Mechanisches bezeichnet, und das, was sich aus jener Materie organisieren soll, bleibt uns ein dunkler unbegreiflicher Punkt. Nun gewann Blumenbach das Höchste und Letzte des Ausdrucks: er anthropomorphosierte das Wort des Rätsels und nannte das, wovon die Rede war, einen nisus formativus, einen Trieb, eine heftige Tätigkeit, wodurch die Bildung bewirkt werden sollte.»

Wir sehen, wie GoETHE mühsam nach den rechten Worten sucht, und 
wir fragen selber: Welchen Charakter hat die der Formbildung zugrundeliegende Tätigkeit der Natur? Hat sie den Charakter einfacher physikalischchemischer Kräfte und Vorgänge, wie wir dieselben am Toten kennenlernen, um sie dann auch in den komplizierten Strukturen des Protoplasmas und des Zellkerns wirksam zu denken? Oder hat diese Tätigkeit den Charakter von Ideen, wie der Mensch sie als Künstler oder als Wissenschaftler erfaßt und sie als Techniker zur Naturbeherrschung auswertet? Sind die Strukturen der lebenden Substanz die Ursachen der Formbildung, oder sind sie Mittel, durch welche angestrebte Formen verwirklicht werden?

GoEthe rät uns 1820 «Bedenken und Ergebung» an. «Wir können bei der Betrachtung des Weltgebäudes in seiner weitesten Ausdehnung, in seiner letzten Teilbarkeit uns der Vorstellung nicht erwehren, daß dem Ganzen eine Idee zum Grund liege, wornach Gott in der Natur, die Natur in Gott, von Ewigkeit zu Ewigkeit, schaffen und wirken möge.»

\author{
«So schauet mit bescheidnem Blick \\ Der ewigen Weberin Meisterstück, \\ Wie ein Tritt tausend Fäden regt, \\ Die Schifflein hinüber, herüber schießen, \\ Die Fäden sich begegnend fließen, \\ Ein Schlag tausend Verbindungen schlägt. \\ Das hat sie nicht zusammengebettelt, \\ Sie hat's von Ewigkeit angezettelt, \\ Damit der ewige Meistersmann \\ Getrost den Einschlag werfen kann.»
}

\title{
Goethe und die wissenschaftliche Medizin seiner Zeit* \\ Von Hans Fischer
}

Es ist nicht meine Absicht, auf Goethes eigene medizinische Studien der Leipziger und Straßburger Studentenjahre, auf die Geschichte seiner Erkrankungen, die ihn behandelnden Ärzte und ihre Behandlungsarten oder auf die physikalisch-diätetische und arzneiliche Selbstbehandlung, bei

* Vortrag, gehalten anläßlich der von der Schweizerischen Gesellschaft für Geschichte der Medizin und der Naturwissenschaften veranstalteten GoEthE-Feier der SNG in Lausanne, 4. September 1949. 
welcher die Brunnenkuren mit und ohne ärztliche Indikation eine entscheidende Rolle spielten, im folgenden einzutreten. Das alles war schon Gegenstand mehr oder weniger eingehender Darstellungen und kann für die früheren Lebensepochen GoETHES in genußreicher Form in «Dichtung und Wahrheit» nachgelesen werden.

Meine Absicht ist vielmehr, Goethes Interesse, nicht an der praktischen Arzneikunst, das ein durchaus laienhaftes war, sondern an der wissenschaftlichen Medizin seiner Zeit dort aufzuzeigen, wo GoEthe mit typischen Vertretern derselben in Berührung gekommen ist. Es sind dies vor allem die Anatomie, von der ich nur die Hirnanatomie stärker hervorhebe, da Goethes eigene humananatomische Studien und der Umkreis ihrer Vertreter hinlänglich bekannt sind, die Physiologie, die Anthropologie, die medizinische Psychologie und die Psychiatrie, wobei ich die weitere Einschränkung mache, daß ich im folgenden auch die physiologische Optik und ihre Vertreter nicht berücksichtige, da hierüber schon genügend Einschlägiges gesagt worden ist.

Haben Goethes Beschäftigung mit der Anatomie des Menschen und mit der Physiologie und Psychologie des Sehens deutlichen Bezug auf seine eigenen wissenschaftlichen Bemühungen und Leistungen, jene zu seiner Typuslehre und den Studien zur vergleichenden Anatomie, diese zu seiner physiologischen Optik und Farbenlehre, so stellen Anatomie und Physiologie des Zentralnervensystems, Psychologie, Anthropologie und Psychiatrie Gebiete dar, welchen GoEThe - der in seiner Naturwissenschaft den geisteswissenschaftlichen Faden nie aus dem Auge ließ - sein besonderes Interesse zuwandte. Bildete doch das Gehirn für den Augen- und Tatsachenmenschen Goethe das «Organ der Seele», das Gefäß sozusagen für alle jene geistig-seelischen Vorgänge, Regungen und Strebungen des Bewußtseins, denen er in Dichtung, Gesprächen, autobiographischen Schriften und Briefen Ausdruck gegeben und als Geistmensch den größten Teil seines unendlich schöpferischen Lebens gewidmet hatte. Neben den naturwissenschaftlichen und autobiographischen Schriften Gozthes, den Aufzeichnungen der Gespräche und der naturwissenschaftlichen Korrespondenz bietet Goethes Bibliothek im Haus am Frauenplan in Weimar, welche auch heute noch - so weit bekannt - zu einem nicht unbeträchtlichen Teil erhalten ist, Anhaltspunkte für den Umfang dessen, was auf den genannten Gebieten an GoETHE herantrat, wobei nicht wenige dieser bibliographischen Zeugen uns auch heute noch davon Zeugnis geben, wieweit GoEthe von ihrem Inhalt Notiz nahm. Meines Wissens sind diese indirekten Zeugen 
von Goethes geistiger Aktivität und umfassender Kenntnis einschlägiger Literatur in solchem Zusammenhang noch nie verwertet worden, so daß ich im folgenden auf diese Quellen an geeignetem Ort jeweils hinweise.

Allgemein bekannt ist Goethes eingehendes Anatomiestudium in den ersten Weimarer Jahren, wobei ihm Professor Loder in Jena kräftig an die Hand ging und ihm durch seinen klaren Vortrag die Kenntnis der menschlichen Anatomie, vorab des Knochen- und Bändersystems, aufs beste vermittelte.

Eines bedeutenden, zu Unrecht fast der Vergessenheit anheimgefallenen Anatomen, Physiologen und Embryologen aus dem Jenenser Kreis, der mit GoEthe in Verbindung stand und von ihm sehr geschätzt wurde, ist hier zu gedenken: Emil Huschkes ${ }^{1}$, Loders Nachfolger auf dem anatomischen Lehrstuhl zu Jena. In Goethes Bibliothek finden sich HuschKes «Beiträge zu Physiologie und Naturgeschichte», 1. Band: «Über die Sinne», Weimar 1824, deren Goethe in der «Metamorphose der Pflanzen» (S. IX) gedenkt.

Weniger bekannt und erst in allerjüngster Zeit in den Vordergrund gerückt durch den Aufsatz Grünthals ${ }^{2}$ ist GoEthes Interesse an der Hirnanatomie.

Sollte nicht das beim Menschen zu so vollkommener Ausbildung gelangte Seelenorgan, das Gehirn, Goethes Interesse besonders fesseln, jenes Organ, welches den Übergang vom Physischen zum Psychischen recht eigentlich bildet, und sollten sich nicht auch in diesem Bereich organischer Bildung, was GoEthe in der ganzen Natur mit Leidenschaft Zeit seines Lebens durch alle Reiche verfolgt hatte, gewisse Gestaltgesetze ergeben, welche sich aus der bei dem einzelnen Menschen verschiedenen Ausbildung

${ }^{1}$ Emil Huschke, geb. Weimar 14. 12. 1797, gest. Jena 19.6. 1858. Huschke war der zweite Sohn des großherzoglichen auch von GoEthe konsultierten Leibarztes in Weimar. Huschke studierte Medizin in Jena, wo er sich mit OKen und dessen naturphilosophischer Richtung befreundete. Er habilitierte sich in Jena mit der Abhandlung: «Über Physiognomik und Mimik». Seine hervorragendsten embryologischen Entdeckungen betreffen die Entwicklung der Schilddrüse (Isis, 1826 und 1828) und die Einstülpung der Linse bei der Bildung des Auges.

Von ihm stammt ferner das Alterswerk «Schädel, Hirn und Seele des Menschen und der Tiere», Jena 1854, das im ganzen naturphilosophische Färbung zeigt, aber gleichzeitig neue Beobachtungen enthält.

2 Ernst Grünthal, Goethes Studien zur Hirnanatomie. In: Abhandlungen zu Goethes Naturwissenschaft, S. 43 (Berner Beiträge zur Geschichte der Medizin und der Naturwissenschaften, Nr. 10. Paul Haupt, Bern 1949). 
des «Seelenorgans» ableiten ließen? Sollten sich nicht irgendwie gesetzmäßige Beziehungen aufdecken lassen, nicht nur zwischen Gesichtsausbildung, Schädelform und seelisch-charakterlichen Eigenschaften, wie in Lavaters Physiognomik, sondern auch im Hinblick auf Schädelgestaltung und Hirnstruktur?

Eine erste Antwort auf diese Frage gab ihm fraglos die Lavatersche Physiognomik, an deren Ausbau Goethe mit jugendlicher Begeisterung mitarbeitete, um sich dann allerdings - weil die gestaltmäßigen Unterlagen und die daraus abgeleiteten Gesetzlichkeiten doch auf zu schwankenden Füßen standen, davon zurückzuziehen.

Und doch sollte GoEthe ein zweites Mal, dreißig Jahre später, diese psychophysische «Morphologie» nun unter bewußter Einbeziehung der Gehirnstruktur in den Gestaltbereich von Schädel, Seele und Geist entgegentreten, in der «Schädellehre» Franz Joseph Galls.

Tief charakteristisch ist für GoEthe, daß er sich auch in diesem Bereich naturwissenschaftlich-psychologischer Bemühungen nicht mit vagen Vorstellungen vom Aufbau des Gehirns begnügte, sondern zu einem wirklichen Verständnis auf Grund exaktester Anschauung zu gelangen suchte, was ihm trotz der Anleitung dazu von seiten der Anatomen Loder ${ }^{3}$, Peter Camper $^{4}$, Soemmering ${ }^{5}$, Vice d'Azyr ${ }^{6}$ u. a. bisher nicht gelungen war.

${ }^{3}$ Justus Christian von Loder, geb. Riga 28. 2. 1753, gest. Moskau 4. 4. 1832. Loder war 1778-1803 Professor der Anatomie, Chirurgie und Hebammenkunst in Jena. Seine Vorlesungen zeichneten sich wie seine Schriften durch große Klarheit aus.

${ }^{4}$ Petrus Camper, geb. Leiden, Mai 1722, gest. Leiden, April 1789. Camper war als Professor der Anatomie, Chirurgie und Medizin in Amsterdam (1763-1773) und als Professor der Anatomie, Chirurgie, theoretischen Medizin und Botanik in Groningen einer der universalsten Gelehrten seiner Zeit.

${ }^{5}$ Samuel Thomas Soemmering, geb. Thorn 25. 1. 1755, gest. Frankfurt am Main 2.3.1830. Studierte in Göttingen Medizin. Schon als Student begann er selbständig über vergleichende Anatomie des Gehirns zu arbeiten. Er promovierte (1778) mit der Abhandlung: «De basi encephali et originibus nervorum cranio egredientium, libri V» durch die er sich sofort einen Namen machte. Goethe suchte Soemmering 1783 erstmals in Kassel auf. In Goethes Bibliothek befinden sich: u. a. S. Th. Soemmering: Vom Hirn und Rückenmark, Mainz 1788 (Widmungsexemplar mit Goldschnitt). GoEtHes Äußerungen hierüber vgl. Grünthal p. 45. S. Th. Soemmering, Vom Bau des menschlichen Körpers. 1791 und 1801.

Derselbe, «Tabulae baseos encephali», Frankfurt 1799, Fol. und «Über das Organ der Seele», Königsberg 1796.

Vgl. auch R. WAGNER, S. Th. Soemmerings Leben und Verkehr mit seinen Zeitgenossen, Leipzig 1844.

${ }^{6}$ Felix Vice d'Azyr, geb. Valognes (Manche) 23. 4. 1748, gest. Paris 20. 6. 1794.1786 be- 
Diese anschauliche Kenntnis vermittelte ihm - und dies spricht für die Ernsthaftigkeit seiner Bemühungen - GALL.

Wer war GALL? Aus der Lebensgeschichte des 1758 in Tiefenbronn in Baden geborenen Franz Joseph Gall nur so viel: er hielt in Wien seit 1786 Privatvorlesungen über seine Schädellehre. Sie wurden 1801 durch kaiserliches Handschreiben wegen «Relegionsgefährlichkeit» verboten und erst später wieder zum Teil gestattet. GALL verließ Wien und hielt 1805 Vorlesungen in Deutschland, Dänemark, Holland und der Schweiz.

Er ließ sich dann 1807 als praktischer Arzt in Paris nieder und eröffnete seine Vorlesungstätigkeit am 15. Januar $1808 \mathrm{mit}$ der «Introduction au cours de physiologie du cerveau» und veröffentlichte mit seinem Mitarbeiter Spurzheim ${ }^{7}$ zusammen die: «Recherches sur le système nerveux en général et sur celui du cerveau en particulier, mémoire présenté à l'Institut de France le 14 mars 1808 suivi d'observation sur le rapport qui en a été fait à cette compagnie par ses commissaires, avec planches» (Paris 1809), in welchen er die französischen Gelehrten von seiner Schädellehre zu überzeugen versuchte. Der Erfolg war eher gering. Sein großes Hauptwerk: «Anatomie et physiologie du système nerveux en général et du cerveau en particulier avec des observations sur la possibilité de reconnaître plusieurs dispositions intellectuelles et morales de l'homme et des animaux par la configuration de leur tête» (Paris 1810-18, 4 volumes) ist dadurch besonders bemerkenswert, daß es umfassende und sehr genaue anatomische Studien über den Faserverlauf im Gehirn enthält. Diese Untersuchungen bildeten für seine Zeit einen bedeutenden Fortschritt und leiten die moderne Hirnanatomie in gewissem Sinne ein.

Außerdem stellte er in diesem Werk seiner «Schädellehre» entsprechend einen genauen Zusammenhang zwischen den einzelnen Geistestätigkeiten und ihrem Sitz in bestimmten Teilen des Gehirns mit der äußeren Schädel-

gann VICQ d'AzYr sein großes anatomisches Werk, von dem nur der erste die Hirnanatomie betreffende, mit prachtvollen farbigen lithographierten Tafeln ausgestattete Band, den GoEthe kannte, unter dem Titel erschienen ist: "Traité d'anatomie et de physiologie, avec des planches colorées représentant au naturel les organes de l'homme et des animaux.» Fol. Paris 1786.

7 Johann Caspar SpUrzheim, geb. 1776 bei Trier, gest. 1832 Boston (USA), war seit 1804 GaLLS Assistent und Mitarbeiter. Später trennte er sich von ihm und trug viel zur Verbreitung der Schädellehre in England bei, von wo sie dann wieder auf den Kontinent zurückwirkte, als GaLl selbst schon ziemlich vergessen war. Von SPUrzherm stammt der Ausdruck «Phrenologie», während GALL selbst nur von Gehirnphysiologie reden wollte. 
form fest. Besondere Aufmerksamkeit wandte er - und darin liegt, wie bei LaVater, die Schwäche seines Systems - auf die äußere Gestaltung des Schädels größten Wert und suchte von dieser aus auf bestimmte psychische und seelische Anlagen und Fähigkeiten zu schließen. Seine Lehre bildet daher eine völlige Parallele zu Lavaters Physiognomischen Fragmenten des Jahres 1775: was dieser hauptsächlich aus den beweglichen Zügen des Gesichts herauszulesen suchte, wollte Gall aus den festeren Formen des Schädels erschließen. Durchgehen wir Goethes (sehr jugendliche) Beiträge zu Lavaters Physiognomischen Fragmenten, so stellen wir fest: Goethe wollte von Anfang an beides, und dies war mit ein Grund zu seinem eingehenden Studium der Anatomie, welches er unter Loders Anleitung in Jena mit so großem Eifer betrieb.

Ist es da zu verwundern, daß GoEthe dreißig Jahre später mit hochgespanntem Interesse in Halle den Vorlesungen und gehirnanatomischen Demonstrationen GaLls im Jahre 1805 folgte, und in langen Gesprächen sich mit ihm über die hirnanatomischen Verhältnisse zu orientieren suchte? Durfte nicht auch mancher hoffen, durch Übersendung von Publikationen, welche die Gallsche Schädellehre betrafen, bei ihm Gehör zu finden?

$\mathrm{Ob}$ dies bei der in Goethes Bibliothek befindlichen Schrift Christian Heinrich Ernst Bischoffs (s. u.): Darstellung der Gallschen Gehirnund Schädellehre, nebst Bemerkungen über die Lehre von Dr. C. W. HufeLAND (Berlin 1805) der Fall war, muß eher zweifelhaft erscheinen, da Bischoff sich in seiner medizinischen Schriftstellerei vollkommen auf dem Boden der Schellingschen Naturphilosophie hielt, in welche er in Jena von Schelling selbst eingeweiht worden war und deren letzte Wandlung in die Offenbarungsphilosophie er mitmachte.

$\mathrm{Da}$ sich Hufeland zur Gallschen Schädellehre zustimmend äußerte, dürfte auch von GoEthe positiv vermerkt worden sein. Ein Gegner GaLls, Jakob Fidelis AckermanN ${ }^{8}$, 1804-5 Professor der Anatomie und Chirur-

8 Jakob Fidelis Ackermann, geb. Rüdesheim, 23. 4. 1765, gest. ebenda 28. 10. 1815. Schüler des Anatomen KarL Kaspar von Siebold d. Ä., studierte seit 1784 in Würzburg, dann in Mainz; später war er Schüler von P. Frank, Scarpa, Volta u. a. in Pavia und wurde 1796-1798 Professor für Anatomie als Nachfolger von SoEMMERING in Mainz, 1804 Professor der Anatomie und Chirurgie in Jena als Nachfolger Loders, 1805 Professor der Anatomie in Heidelberg.

Einige seiner in Jena verfaßten medizinischen Abhandlungen sind in GoEthes Bibliothek gelangt; offenbar Dedikationen des in amtlicher Stellung Untergebenen an den die «Oberaufsicht» führenden Staatsminister von GoETHE, obgleich dieser mit den medizinischen Anstalten nicht direkt zu tun hatte. 
gie zu Jena, der Galls Vorträge in Jena gehört haben dürfte, verfaßte eine ebenfalls in Goethes Bibliothek befindliche Gegenschrift, die von GaLL mit dem eben dort befindlichen Aufsatz: Franz Joseph Gall, «Beantwortung der AcKermannschen Beschreibung und Widerlegung der GaLLschen Hirn- und Schädel- und Organenlehre vom Gesichtspunkte der Erfahrung» (Halle 1806), beantwortet wurde.

Der auf dem Gebiet der Hirnanatomie und der Psychiatrie tätige ReIL (s. u.) war GALL zunächst wohlgesinnt. Er erklärte, daß er «in GALLS anatomischen Demonstrationen mehr gesehen habe, als er geglaubt hätte, daß ein Mensch in seinem ganzen Leben entdecken könne» (zit. bei MöвıUs', p. 101). Die Angriffe Ackermanns in Jena und Walters in Berlin bewirkten, daß REIL, der seine Gehirnuntersuchungen (Insula REILI!) auf GaLlS Antrieb vorgenommen hatte, ihn in schmählicher Weise (1806) angriff, was GoEthes Zorn erregte. Er ging darin so weit, die Kreuzung der Pyramiden u. a. gehirnanatomische Feststellungen zu leugnen. Dabei vertraten sowohl Reil wie Ackermann in ihrer Nerven- und allgemeinen Naturlehre völlig abstruse naturphilosophische Ideen.

Mehr in das Reich der physischen Anthropologie, an deren Entwicklung Goethe großen Anteil nahm, führen uns die vom befreundeten vergleichenden Anatomen Johann Friedrich Blumenbach ${ }^{10}$ fortlaufend zugestellt erhaltenen, im Laufe von etwa dreißig Jahren erschienenen Beschreibungen von Schädeln verschiedener Völker und Rassen, deren erste «Decas collectionis suae craniorum diversarum gentium illustrata» 1790, wie deren letzte 1828 in Göttingen herauskam. Die ganze Serie findet sich in Goethes Bibliothek. An diesem konkreten Material mußten sich Beobachtungen anstellen lassen, welche auf Lavaters Physiognomik ebenso hinwiesen wie auf Galls Phrenologie, zunächst aber doch ein anderes gemeinsames Interesse Blumenbachs und Goethes berührten: die im Werden begriffene physische Anthropologie.

Wie stellte sich Goethe selbst zu der Schädellehre Galls? Goethe ist dreißig Jahre nach den Erfahrungen mit Lavater, als ihm GaLL und seine Lehre erstmals entgegentrat, vorsichtig geworden, bei aller Sympathie, die er zu solchen auf Gestaltbeziehungen aufgebauten Lehren im Grunde hatte.

${ }^{9}$ P.J.Möвıus, Franz Joseph Gall, Leipzig, 1905.

${ }^{10}$ Johann Friedrich Blumenbach, geb. Gotha 11. 5. 1752, gest. Göttingen 22. 1. 1840. Von 1776 Professor der Medizin in Göttingen bis zu seinem Tode. Er gilt als der Begründer der neuzeitlichen Anthropologie. Vgl. K. Fr. H. MARX, Zum Andenken an Blumenbach, Göttingen 1840. 
Über Galls Schädellehre hat Goethe wie Grünthal, entgegen Möвius, nachwies, stets zurückhaltend geurteilt, während er den hirnanatomischen Entdeckungen GaLLS begeistert zustimmte, die von dem hervorragenden Hirnanatomen REIL, von dem noch zu sprechen sein wird, abgelehnt wurden! Goethes Tatsachensinn hatte wieder einmal recht. Wir anerkennen heute, wo wir mit größerer innerer Freiheit und Distanz der Gallschen Lehre gegenüberstehen, das wirklich positive und bedeutende derselben, nämlich die Tatsache, daß sich aus der hirnanatomischen Grundlegung GaLlS die moderne Hirnanatomie und Hirnpathologie teilweise entwickelt hat!

Was wir ablehnen an GaLLS Schädellehre, ist seine Behauptung, daß besonders entwickelte Hirnorgane jeweils äußerlich an der Schädelbildung erkennbar seien. Schon GoEthe nannte 1803 dies eine «wunderliche Lehre, der es denn doch so wenig als der Lavaterschen, an einem Fundament fehlen möchte».

Was ihm aber GALL - erstmals in seinem Leben vermittelte - und was GoEthe in Begeisterung versetzte, war eine anschauliche klare Ansicht vom Aufbau des menschlichen Gehirns. Zwar kannte Goethe Vicq d'Azyrs herrliche «Planches Anatomiques du cerveau de l'homme», aber, was er dort fand, war kein Überblick, waren «Lokalkenntnisse», «weshalb auch noch vor kurzem», wie GoETHE sagte, «die schönen Abbildungen von VICq d'AzYr mich völlig in Verzweiflung gesetzt hatten»».

Was ihm weder Vice d'AzYr noch der befreundete Frankfurter Anatom Samuel Thomas Soemmering mit seinen vortrefflichen Abhandlungen und Tafeln zu vermitteln vermochten, gab ihm GALL, dessen Vorlesungen Gozthe 1805 in Halle so begierig gefolgt war. - Hier fand er den «ariadneischen Faden», den er trotz eingehendster Bemühungen, mit der Hirnanatomie ins klare zu kommen, bis anhin vermißt hatte. - Bedeutete es nicht eine Art Huldigung, wenn Goethe den Abguß von Galls Schädel verlangte, nachdem Gall schon früher einen Abguß von GoEthes Haupt - den einzigen, von Weißer verfertigten, den GoEtre zuließ - veranlaßt hatte.

Wie unabhängig sich Goethe von Theorienbildungen, welche die Beziehungen zwischen dem Gehirn als dem Organ der Seele und der Lokalisation der Seelenfunktionen im Zentralnervensystem hielt, zeigt seine kritische Einstellung, ja eigentlich scharfe Ablehnung von Soemmerings Theorie vom liquor cerebrospinalis als dem «sensorium commune» der seelischen Emanationen, welchen Soemmering an Stelle der Zirbeldrüse Des- 
CARTeS' als dem Sitz der Seele, des centrum ovale Vieussens', des corpus striatum von Thomas Willis, des Kleinhirns Drelincourts', des Pons («Hirnknoten») Hallers und Wrisbergs u. a. setzte; - eine Kritik am spekulativen, nicht am anatomischen Teil, die KANT, dem das sonst so schöne Werk Soemmerings «Über das Organ der Seele» (1796) gewidmet war, teilte.

Dabei ging die persönliche Bekanntschaft und Freundschaft GoEthes mit Soemmering schon auf viele Jahre zurück und setzte sich unvermindert fort. Soemmering hatte Goethe auch sein Büchlein «Vom Hirn und Rückenmark» (1788) in einem schönen mit Goldschnitt versehenen Widmungsexemplar überreicht, so wie es noch in GoEthes Privatbibliothek zu finden sein dürfte. Sein «Organ der Seele» (1796) sandte Soemmering ebenfalls an Goетнe, der den Empfang am 25.Juni 1796 bestätigte, das Werk aber erst in einem Brief vom 28. August 1796 in der genannten Art ausführlich besprach und kritisierte. Und ferner finden wir in GoEthes Besitz eine Frankfurter Folioausgabe des Jahres 1799 von Soemmerings berühmten «Tabulae baseos encephali», in denen die anatomischen Verhältnisse der Hirnbasis zu anschaulicher Darstellung gelangten.

Goethes Beziehungen zur Gehirnanatomie seiner Zeit sind damit nicht erschöpft. Wie weit allerdings Goethe Karl Friedrich Burdachs ${ }^{11}$, des Königsberger Anatomen berühmtes Werk: «Vom Baue und vom Leben des Gehirns» (3 Bände, Leipzig 1819-26), in welchem seine Entdeckung der nach ihm benannten sensiblen Hinterstrangbahn beschrieben ist, zu schätzen wußte, dessen erster Band (Leipzig 1819) sich in GoEthes Bibliothek befindet, dürfte kaum noch festzustellen sein. (Die ersten 157 Seiten des Buches sind aufgeschnitten.) Burdach hat in diesem bedeutenden Werk eine sehr gewissenhafte Geschichte der Gehirnanatomie geliefert, in welcher er unter den deutschen Gelehrten des 19. Jahrhunderts, welche wirkliche Fortschritte gebracht haben, GaLL (nach heutiger Auffassung

${ }^{11}$ Karl Friedrich Burdach, geb. Leipzig 12.6. 1776, gest. Königsberg 16. 7. 1847. Burdach studierte Medizin in Leipzig und stand anfangs stark unter dem Einfluß ScHELLINGs («Asclepias und John Brown, eine Parallele», Leipzig 1800). 1811 wurde er zum Professor für Anatomie, Physiologie und Gerichtliche Medizin in Dorpat ernannt, 1814 zum Professor für Anatomie und Physiologie in Königsberg, wo er die dortige anatomische Anstalt gründete und mit RATHKe und K. E. von BAER die anatomische Sammlung anlegte. Er interessierte sich vorwiegend für Entwicklungsgeschichte und das Gehirn («Beiträge zur näheren Kenntnis des Gehirns», Leipzig 1805). Burdachs größtes Verdienst liegt in der zusammenfassenden Darstellung der Physiologie seiner Zeit in dem Werk: «Die Physiologie als Erfahrungswissenschaft». 6 Bände, Leopold Voss, 1826-1840. 
zweifellos mit Recht) an die Spitze stellt. Wie sehr BuRdach recht hatte, beweist die Einstellung JACQUES LoEBS, der in seiner «Vergleichenden Gehirnphysiologie» (1890) (zit. n. Möвıus, p. 114) schreibt: «Die moderne Funktionslokalisation im Großhirn ist historisch die direkte Fortsetzung der Gallschen Phrenologie.» Ein ebenfalls berühmtes Werk Burdachs, dessen stark naturphilosophische Einstellung Schellingscher Richtung offensichtlich ist, das er ebenfalls Goethe zustellte «Über die Aufgabe der Morphologie» (Leipzig 1817), dürfte nicht nur dem Titel nach GoEthes Interesse beansprucht haben. «Morphologie», dieser von GoETHE selbständig geprägte Ausdruck, wurde von BURDAGH in ähnlichem Sinne verwendet, bevor Goethes Schriften zur Morphologie veröffentlicht waren. Eine bedeutende und wenig bekannte Anregung gab BurdacH durch sein Buch: «Über Psychologie als Naturwissenschaft» (Berlin 1828), in welchem er die vergleichende Beobachtung der tierischen Seelenäußerungen empfahl, ein Postulat, das erst in unserer Zeit Verwirklichung gefunden hat.

Unmittelbar berührt sich GoEthes Interesse an der Hirnanatomie und -physiologie mit seinen psychologischen und psychiatrischen Erfahrungen ${ }^{12}$.

Der Goethe bestbekannte ärztliche Psychologe und Freund war CarL Gustav Carus ${ }^{13}$, dessen Verdienste um die Entwicklung der Psychologie und Typenlehre als Wissenschaft zu bekannt sind, als daß hierüber näheres auszuführen wäre. Ein schönes Widmungsexemplar mit Goldschnitt seiner «Vorlesungen über Psychologie, gehalten im Winter 1829-30 zu Dresden» (Leipzig 1831) gehört Goethes Bibliothek an. Von den mannigfaltigen Beziehungen zwischen Goethe und Carus, der in seiner Naturauffassung stark unter Goethes Einfluß stand, legt sein Goethe-Buch ${ }^{14}$

${ }^{12}$ Vgl. auch: L. KLAGES, Goethe als Seelenforscher. 1932.

${ }^{13}$ Carl Gustav Carus, geb. Leipzig 3. 1. 1779, gest. Leipzig 7. 7. 1868. Carus promovierte in Leipzig 1811, habilitierte sich noch im gleichen Jahr für vergleichende Anatomie ebenda und wurde 1814. Professor der Entbindungskunst an der medizinisch-chirurgischen Akademie zu Dresden (bis 1827). Seine Bedeutung für die vergleichende Anatomie und für die Anthropologie, besonders aber für die Psychologie, ist viel größer als für die Medizin. Unter seinen anthropologischen (ziemlich spekulativen) Veröffentlichungen ist beispielsweise zu nennen: C. G. CARUS, Grundzüge einer neuen und wissenschaftlich begründeten Cranioskopie (Schädellehre). Stuttgart 1841.

${ }^{14}$ C. G. Carus, Goethe, zu dessen näherem Verständnis, 1843. Neu herausgegeben im Verlag E. Rentsch, Zürich 1949.

- - Goethe, dessen Bedeutung für unsere und die kommende Zeit. Wien, W. Braumüller, 1863.

Vgl. auch: Cr. Burckuard, Carus als Psychologe, E. Diederichs, Jena 1927. 
beredtes Zeugnis ab. Hier zu bemerken ist, daß Carus als Gegner Galls auftrat, was vielleicht damit zusammenhängt (nach MöвıUs, p. 124), daß er seine eigene Lehre mit derjenigen GaLLS und mit der Lehre von den drei Schädelwirbeln und der «Dreiteilung der Seele» vermengte.

In seinem letzten Lebensjahr erhielt GoETHE auch das hauptsächlich hirnanatomische Werk des Psychiaters Gottlieb Heinrich Bergmann ${ }^{15}$, «Neue Untersuchungen über die innere Organisation des Gehirns, als Beitrag zu einer Grundlage der Psychologie und Pathologie desselben», Hannover 1831, zugestellt, das von der Fachwelt, wie auch seine «Untersuchungen über die Struktur der Mark- und Rindensubstanz des großen und kleinen Gehirns»(Müllers Archiv für Physiologie, Jg. 1841), weil in der Interpretation auf der Pneumalehre basierend, als zu phantastisch abgelehnt wurde. Bemerkenswert sind diese Untersuchungen dennoch, weil sie das Bestreben der damaligen Psychiatrie zeigen, durch konkrete hirnanatomische Feststellungen die psychiatrischen Befunde zu stützen.

Weniger bekannt dürfte die sehr positive Einstellung Goethes zu dem Psychologen Ernst Stiedenroth ${ }^{16}$ sein, dessen «Psychologie zur Erklärung der Seelenerscheinungen, erster Teil» (Berlin 1824) Goethe in seiner Zeitschrift «Zur Morphologie» (Band 2), als «glückliches Ereignis» im Sinne einer hoch-willkommenen Begegnung besprach. In welchem Sinn diese hohe Anerkennung Stiedenroths gemeint war, sagt Goethe im gleichen Band seiner Zeitschrift, in dem «Tibia und Fibula» benannten Abschnitt seiner «Vergleichenden Knochenlehre» (S. 177).

«Ein Mann, wie Hr. Ernst Stiedenroth, sollte seine erlangte hohe Einsicht in die Functionen des menschlichen Geistkörpers und Körpergeistes treulich anwenden, um die Geschichte irgend einer Wissenschaft zu schreiben, welche denn symbolisch für alle gelten würde.»

Das Einheitsstreben GoEthes ist auch auf diesem Gebiet nicht zu verkennen. Was er hier, im Reiche des Geistes «symbolisch» nannte, war es nicht das Streben nach der Erkenntnis des «Urphänomens», dessen Existenz er auch im Geistigen anerkannte?

${ }^{15}$ Gottlieb Heinrich Bergmann, geb. Erichshagen bei Nimburg an der Weser 12. 6. 1781, gest. Hildesheim, 29.10.1861. Studierte Medizin in Göttingen, promovierte daselbst 1804 . War eine Zeitlang an Zuchthaus und Irrenanstalt zu Celle angestellt, seit 1832 Direktor der 1827 gegründeten Irrenheil- und Pflegeanstalt im St.-Michaelis-Kloster in Hildesheim.

${ }^{16}$ Heinrich Ernst Stiedenroth, geb. Hannover 11.5.1794, gest. Greifswald 3. 5. 1858. Scharfsinniger Philosoph aus der Herbartschen Schule. Seine schriftstellerische Hauptleistung ist die genannte «Psychologie». 
Zu den psychologisch orientierten Arztfreunden Goethes gehört einer der berühmtesten und verehrtesten Ärzte seiner Zeit, dessen Vater und Großvater Leibärzte am Weimarischen Hof gewesen waren: Christian Wilhelm Hufeland ${ }^{17}$, der mit Wieland, Herder, Goethe und Schiller, dessen Arzt er war, vertrauten Umgang hatte. Der romantischen Medizin eines John Brown und Franz Anton Mesmer als einer unwissenschaftlichen Medizin abgeneigt ${ }^{18}$, und von diesen Kreisen deshalb heftig angegriffen, gehörte HUfELANd zu jenen Ärzten, wie etwa Tissot in Lausanne, welche strenge Wissenschaftlichkeit mit Allgemeinverständlichkeit zu verbinden wußten und dadurch ungeheuren Einfluß gewannen. Als Professor in Jena hatte Hufeland in seinen Vorlesungen bis zu 500 Hörer. Von seiner in alle Sprachen übersetzten «Kunst das menschliche Leben zu verlängern» (1796) oder seiner «Makrobiotik» (1805), wie er das Buch später nannte, einer Diätetik der Lebensführung, wußte auch GoEthe Gebrauch zu machen.

Eine Vorstudie dazu: «Gemeinnützige Aufsätze zur Beförderung der Gesundheit, des Wohlseins und vernünftiger medizinischer Aufklärung», erster Band (Göschen, Leipzig 1794) findet sich in Goethes Bibliothek. Aufgeschnitten ist u. a. der Abschnitt «Gefahren der Einbildungskraft». Ebenso finden sich dort Hufelands : «Ideen über Pathogenie», Jena 1795. Hufeland gehörte zu jenen ausgezeichneten Ärzten, welche den psychischen Anteil an Krankheit und Gesundheit des Menschen viel stärker betonten, als dies (leider) gemeinhin üblich ist. In einem Briefwechsel

${ }^{17}$ Christoph Wilhelm Hufeland, geb. Langensalza (Thüringen) 12. 8. 1762, gest. Berlin 25. 8. 1836. Studierte von 1780 an Medizin in Jena und Göttingen, wo er 1783 zum Doktor der Medizin promovierte. Nach Weimar zurückgekehrt, mußte er sofort die Praxis seines fast erblindeten Vaters übernehmen. 1793 wurde Hufeland von Großherzog CarL August als Professor der Medizin nach Jena berufen. Nach Entdeckung der Kuhpockenimpfung durch EDward Jenner (1796) setzte er sich sofort für sie ein. 1800 wurde Hufeland als erster Arzt der Charité und königlicher Leibarzt nach Berlin berufen. Wie schon früher wurde er auch hier durch die Brownianer aufs heftigste angegriffen. An der Gründung der Berliner (HuмвоцDT-) Universität war HufELAND stark beteiligt und wurde 1810 zum Professor für spezielle Pathologie und Therapie an derselben ernannt. Im Zusammenhang damit eröffnete er eine medizinische Poliklinik, das erste Institut dieser Art für arme Kranke.

Der GALLschen Schädellehre widmete er die schon genannte eingehende Darstellung und Beurteilung.

${ }^{18}$ Sein erster literarischer Versuch betraf: «MEsmer und sein Magnetismus» (Deutscher Merkur 1785). 
zwischen Goethe und Hufeland ${ }^{19}$, in welchem der Einfluß der barometrischen Luftveränderungen auf das Leben organischer Wesen zur Sprache kommt, drückt sich diese psychologische Einstellung HufELAnds charakteristisch aus.

Wenn wir im folgenden auf einige Psychiater aufmerksam machen, mit denen GoEthe teils in direktem persönlichen Verkehr stand, teils mit ihnen korrespondierte oder deren Werke ihm teilweise bekannt waren, erscheint es wohl notwendig, auf die Hauptströmungen der Psychiatrie seiner Zeit, soweit sie Ärzte und Psychiater betreffen, die in den Gesichtskreis GoEthes traten, kurz einzugehen.

Voranzustellen ist als Vorläufer einer neuen Zeit der im Zusammenhang mit Gall bereits genannte Gehirnanatom, Physiologe und Psychiater Johann Christian Reil ${ }^{20}$, ein genialer Arzt und Hauptvertreter des "Vitalismus» in Deutschland, den er in der berühmt gewordenen Abhandlung «Von der Lebenskraft» $(1796)^{21}$ vertrat. ReIL wollte aber vor allem der Medizin durch enge Verbindung mit der Physiologie wissenschaftliche Grundlagen geben. Bahnbrechend sind ReILs unter dem Einfluß Galls entstandene Arbeiten über den Bau des Nervensystems, besonders die «Fragmente über die Bildung des kleinen Gehirns im Menschen» (1807-8), mit der nach ihm benannten Insula ReILI.

Große Verdienste hat sich ReIl um die Förderung der Psychiatrie erworben. Wenn auch seine Bemühungen um die Gründung von psychiatrischen Instituten in Halle und Berlin nicht erfolgreich waren, gab er doch wichtige Anregungen dazu, welche schon die nächste Generation verwirklichte.

Mit seinen anatomischen Arbeiten über die Struktur des Gehirns und

${ }^{19}$ F. Th. Bratranek, Goethes naturwissenschaftliche Korrespondenz (1812-1832). Im Auftrage der von Goetheschen Familie herausgegeben. 2 Bände. F. A. Brockhaus, Leipzig 1874.

${ }^{20}$ Johann Christian Reil, geb. Rhaude (Ostfriesland) 20. 2. 1759, gest. Halle 22. 11. 1813. Studierte Medizin in Göttingen und Halle und promovierte daselbst 1782. Schon bald darauf zum Professor der inneren Medizin und Direktor der Klinik in Halle ernannt, war sein Ruf bald so bedeutend, daß er 1810 den Lehrstuhl der klinischen Medizin an der neu gegründeten Universität Berlin erhielt. Während des Krieges 1813 hatte er die oberste Leitung der Kriegsspitäler am linken Elbeufer unter sich; er starb in dieser Tätigkeit am Typhus.

${ }^{21}$ In ReILs Archiv für Physiologie, Bd. 1. 
mit seiner zeitweise großen Sympathie für GaLls Schädellehre verband sich eine besondere Vorliebe für psychiatrische Studien ${ }^{22}$.

In seiner klassisch gewordenen Schrift: «Rhapsodien über die Anwendung der psychischen Kurmethode auf Geisteszerrüttungen» (Halle 1803), welche er auch GoETHE übermittelte ${ }^{23}$, gab er eine packende Schilderung von dem erbärmlichen Zustand der deutschen Irrenhäuser und trug vieles zur Besserung und auch zur Anwendung physischer Heilmittel bei Geisteskranken bei ${ }^{24}$.

Wenn wir von der romantischen oder naturphilosophischen Richtung der Medizin und Psychiatrie absehen, der ein KIeser ${ }^{25}$, BischofF ${ }^{26}$ und

${ }^{22}$ In einem Brief Alexander von Humboldts an Goethe vom 16. Juli 1795 empfiehlt ihn Humbold an Goethe angelegentlich. Vgl.: F. Th. BratraneK, Goethes Briefwechsel mit den Gebrüdern Humboldt (1795-1832). Im Auftrage der von Goetheschen Familie herausgegeben. Leipzig, F. A. Brockhaus 1876.

${ }^{23}$ In Goethes Bibliothek findet sich: ReIL Und Kayssler, Magazin für die psychische Heilkunde. 1. Bd., Heft 1-3, 1805-6; darin im 1. Heft die «Rhapsodien».

${ }^{24} \mathrm{Daß}$ er auch psychische Heilmethoden anwandte, zeigt $u$. a. folgende interessante Stelle: «Erregungen der Seele sind die Mittel, durch welche die Intemperatur der Vitalität des Gehirns im Wahnsinn rectifiziert werden muß.»

${ }^{25}$ Dietrich Georg Kieser, geboren Harburg (Hannover) 24. 8. 1779, gestorben Jena 11. 10. 1862. KIESER war einer der Hauptvertreter der naturphilosophischen Richtung in der Medizin und ein eifriger Anhänger des Mesmerismus, als Praktiker aber durchaus auf realem Boden stehend. Er promovierte in Göttingen 1804 mit einer naturphilosophischen Dissertation. 1812 wurde er zum Professor der Medizin in Jena und zum Brunnenarzt in Berka ernannt. Das Bedürfnis nach weiterer Ausbildung führte ihn nach Paris und Leiden. Als Oberstabsarzt hatte er 1818 die Kriegsspitäler in Lüttich und Versailles unter sich. - Er vertrat die Universität Jena auf dem weimarischen Landtag und trug in dieser Stellung zur Verbesserung des Medizinal- Gefangenen- und Irrenheilwesens bedeutendes bei. 1846 wurde KIESER auch zum Direktor der Irrenheil- und Pflegeanstalt in Jena ernannt; er übernahm 1847 gleichzeitig die psychiatrische Klinik und eine Privatheilanstalt für Geisteskranke («Sophronisterium») und widmete von da an seine Zeit vorwiegend den Geisteskranken. Seine psychiatrischen Schriften zeigen reiche Erfahrung auf dem Gebiet der Geisteskrankheiten, die er als somatische auffaßte und behandelte.

${ }^{26}$ Christian Heinrich Ernst Bischoff, geb. Hannover 14. 9. 1781, gest. Bonn 5. 3. 1861. 1818-1861 Professor der Pharmakologie und Staatsarzneikunde an der neugegründeten Bonner Universität. Seine positive Einstellung zu GALL wurde bereits erwähnt; sie steht wie seine «Lehre von den chemischen Heilmitteln» (Bonn 1825-1831) vollkommen auf dem Boden Schellingscher Naturphilosophie, in welche er durch Schelling selbst während seines Jenaer Medizinstudiums eingeweiht worden war. 
Schelling ${ }^{27}$, die Anhänger des tierischen Magnetismus mit Mesmer, Eschenmeyer, Passavant ${ }^{28}$ und alle jene etwas dunklen Richtungen des medizinischen Subjektivismus und Sensualismus, insbesondere eines JоHN Brown ${ }^{29}$, angehörten und denen Novalis ${ }^{30}$ in seinem zarten Denken so viel Gewicht beimaß, sind in der deutschen Psychiatrie zwei Hauptrichtungen zu erkennen: die somatische und die psychische. Unter den Anhängern der somatischen und den Verfechtern der psychischen Auffassung der Geisteskrankheiten entbrannte ein Streit, welcher die vier ersten Dezennien des 19. Jahrhunderts beherrschte.

Die psychische Richtung sah die unmittelbare Ursache der Geisteskrankheiten von der Seele selbst ausgehen und betrachtete die Verleugnung von Vernunft und Moral, die Leidenschaften und die Sünde als die Quellen der psychischen Krankheiten. Die gleichzeitig auftretenden somatischen Störungen sind nach dieser Auffassung sekundär bedingt, d. h. durch das Leiden der Seele erzeugt.

Dieser Standpunkt wurde am konsequentesten von HeInroth, BeneKe und Ideler vertreten, von denen nur Heinroth in Goethes Gesichtskreis getreten ist.

Неinroth hat durch seine Vorlesungen und Publikationen (z. B. durch sein «Lehrbuch der Störungen des Seelenlebens», Leipzig 1818, und sein «Lehrbuch der Seelengesundheitskunde», Leipzig 1823) zur psychologischen Begründung der Psychiatrie wesentliches beigetragen.

Wir dürfen annehmen, daß dem Geistmenschen GoEthe diese Richtung der im Werden begriffenen deutschen Psychiatrie sympathisch war und daß

${ }^{27}$ F. W. J. Schelling gab mit dem Brownianer A. F. Marcus die «Jahrbücher der Medizin als Wissenschaft», Tübingen in der Cottaschen Buchhandlung heraus, von denen des «Ersten Bandes erstes Heft» (I-XX und 1-206), erschienen 1805, sich in GoEtHes Bibliothek befindet. Im ganzen erschienen 3 Bände 1805-1808.

${ }^{28}$ Vgl. Werner Leibbrand, Romantische Medizin, Goverts Hamburg 1937, und Ricarda Huch, Die Romantik, 2 Bände, Haessel, Leipzig, 1922.

${ }^{29}$ JoHn Brown (1735-1788). Mit seiner Krankheitslehre, die er in den «Elementa medicinae» (1780) ausführlich zur Darstellung brachte, erregte Brown nicht nur die Aufmerksamkeit der englischen Ärzte und eines weiteren Publikums, sondern eroberte in kurzer Zeit auch den Kontinent. 1801 kam GoETHE mit dieser Lehre erstmals praktisch in Berührung. Er befürchtete, wie die Tag- und Jahreshefte 1801 erkennen lassen, die Pyrmonter Trinkkur könnte bei ihm einen zu starken «contrastimulus» auslösen. Im weitern aber war er der Lehre nicht besonders zugetan oder lehnte sie ab.

${ }^{30}$ H. Fischer, Die Krankheitsauffassung Friedrich von HARdenbergs (Novalis) (1772 bis 1801), ein Beitrag zur Medizin der Romantik. Verh. Naturf. Ges. Basel 56, 390 (1945). 
er Johann Christian August Heinroths ${ }^{31}$ Werke, Professors der psychischen Heilkunde an der Universität Leipzig, mit Interesse verfolgte. Außer Frage steht, daß er sich für Неinroths Bemühungen, die Anthropologie in die Medizin einzuführen, interessierte. Heinroth war schon in seiner Leipziger Habilitationsschrift «Über das Bedürfnis des Studiums der Anthropologie» (1806) für dieses Fach eingetreten. Später übermittelte er Goethe sein «Lehrbuch der Anthropologie» (Leipzig 1822), das sich vollständig aufgeschnitten in GoEthes Bibliothek befindet. Von diesem handelt Goethe im 2. Band «Zur Morphologie» ausführlich unter dem bemerkenswerten Titel: «Bedeutende Fördernis durch ein einziges geistreiches Wort». Heinroth analysiert darin Goethes Wesen und bemerkt vor allem, daß Goethes Denkvermögen gegenständlich tätig sei, daß sein Anschauen selbst ein Denken, sein Denken ein Anschauen sei. Wozu GoEthe bemerkt: «Wend' ich mich um zu dem gegenständlichen Denken, das man mir zugesteht, so find' ich, daß ich eben dasselbe Verfahren auch bei naturhistorischen Gegenständen zu beobachten genötigt war. Welche Reihe von Anschauung und Nachdenken verfolgt' ich nicht, bis die Idee der Pflanzenmetamorphose in mir aufging, wie solches meine ,Italienische Reise ${ }^{6}$ den Freunden vertraute.»

Und weiter: «Was nun von meinem gegenständlichen Denken gesagt ist, mag ich wohl auch ebenmäßig auf eine gegenständliche Dichtung beziehen. Mir drückten sich gewisse große Motive, Legenden, uraltgeschichtlich Überliefertes so tief in den Sinn, daß ich sie vierzig bis fünfzig Jahre lebendig und wirksam im Innern erhielt.»

Für Goethe bildete des Psychiaters Heinroth treffende Charakterisie-

31 Johann Christian August Heinroth, geb. Leipzig 17. 1. 1773, gest. ebenda 26.10.1843. Неinrotн war seit 1814 Arzt am Zucht-, Waisen- und Versorgungshaus zu St. Georgen in Leipzig, das auch Geisteskranke beherbergte. Sein Werk «Beiträge zur Krankheitslehre» brachte ihm den Lehrstuhl für psychische Therapie in Leipzig ein; 1827 wurde er Ordinarius und trat 1830 in die medizinische Fakultät ein. Seine Vorlesungen über Anthropologie und Psychiatrie waren sehr geschätzt. Unter den Werken ist noch sein «System der psychisch-gerichtlichen Medizin» (Leipzig 1825) zu nennen.

Heinnoth ist der Hauptvertreter der rein psychischen Auffassung der Geisteskrankheiten, um nicht zu sagen, der philosophischen Richtung der Psychiatrie, wie etwa folgendes Zitat aus seiner Seelenheilkunde zeigt: «In dem Selbstgefühl des Nicht-sichselbst-Angehörens besteht das Wesen der Melancholie; das Gebundensein an verkehrte Weltbegriffe, wie sie dem Interesse des Individuums zusagen, macht das Wesen des Wahnwitzes aus; das Wesen der Narrheit besteht in dem Haften und Hangen an sich selbst als einer vorzüglichen Person», usw. 
rung seiner Geistesart eine Selbsterhellung, welche er dankbar als Bereicherung empfand und ihr entsprechenden Ausdruck gab.

Die somatische Richtung in der deutschen Psychiatrie faßte die Störungen des Seelenlebens lediglich als Ausfluß leiblicher Zustände auf. Die Seele scheine, lautet diese Theorie, nur in den Äußerungen ihrer Funktionen alieniert, weil das Somatische, an welches ihre Tätigkeit gebunden ist, erkrankt sei. Es gebe keine selbständigen psychischen Krankheitsformen, sondern diese dürfen nur als Symptome körperlicher Krankheiten angesehen werden.

Bedeutende Vertreter dieser Richtung waren Nasse und Jacobi. ChriStian Friedrich Nasse ${ }^{32}$, als Schüler Reils, suchte den Ausgangspunkt aller psychischen Krankheiten in körperlichen Zuständen. Er betonte, daß bei allen geistigen Störungen das Gehirn erkrankt sein müsse. Sein Hauptaugenmerk war auf die Erforschung der Beziehungen zwischen seelischen und körperlichen Krankheitserscheinungen gerichtet.

Nasse sandte Goethe mit einem Begleitbrief, datiert 17. Mai $1825^{33}$, die ersten Hefte des GoETHE gewidmeten Jahrganges der von NASSE gegründeten «Zeitschrift für Anthropologie»34. Die Antwort Goethes ist nicht

82 Christian Friedrich Nasse, geb. Bielefeld 18. 4. 1778, gest. Bonn 18. 4. 1851. Nasse studierte seit 1797 in Halle und Berlin als Lieblingsschüler ReILs und promovierte hier 1800. Einige Zeit war er praktischer Arzt in Bielefeld, Göttingen und Weimar. 1815 wurde er Professor der Medizin und Direktor des neuen klinischen Institutes in Halle, 1819 kam er in gleicher Stellung nach Bonn.

NASSES Hauptverdienst liegt in der Pflege der physiologischen Richtung der Medizin. Er war der erste deutsche Arzt, der die physikalische Diagnostik am Krankenbett übte. Als einer der ersten wandte er das Mikroskop in den klinischen Vorlesungen an. Im weiteren erwarb er sich große Verdienste um die Entwicklung der Psychiatrie und des psychiatrischen Unterrichts in Deutschland.

Zahlreiche Aufsätze im Archiv für tierischen Magnetismus entstammen seiner Feder. Außerdem gab er die «Zeitschrift für psychische Ärzte» (1818-1828) und mit JACовI die «Jahrbücher für Anthropologie und zur Pathologie und Therapie des Irreseins» (1831) heraus. Es ist bemerkenswert, daß schon in diesen Jahren Anthropologie und Psychiatrie in engem Zusammenhang auftreten, wie dies dann erst wieder durch Cesare Lomвroso bewußt geschah.

Weiterhin war NASSE der Herausgeber der «Zeitschrift für die Beurteilung und Heilung krankhafter Seelenzustände» (1838).

${ }^{33}$ F. Th. BratraneK, Goethes naturwissenschaftliche Korrespondenz, Bd. 2, p. 1.

${ }^{34}$ «Zeitschrift für die Anthropologie» in Verbindung mit andern herausgegeben von Friedrich Nasse. Erstes Vierteljahrsheft für 1825; auf starkem Papier, Goethe gewidmet. Ebenso Heft 2-4, 1825. 
erhalten. In Goethes Bibliothek findet sich von Nasses Schriften außerdem das von C. A. Eschenmeyer, D. G. Kieser und Friedrich Nasse herausgegebene «Archiv für tierischen Magnetismus» (1. und 2. Band, 1817-8), über das sich GoEtHE meines Wissens nirgends geäußert hat, wohl aber über den tierischen Magnetismus.

Mit Karl Wigand Maximilian Jacobi ${ }^{35}$, jüngstem Sohn des Goethe früher nahestehenden Philosophen Friedrich Heinrich Jacobi treten wir näher in den Kreis der praktischen Irrenpflege ein. JAсовI wurde 1793 als Jenenser Medizinstudent mit GoEthe bekannt, der ihn häufig zu sich zog und seine anatomischen Kenntnisse mit ihm auffrischte. In diesem $\mathrm{Zu}$ sammenhang interessiert uns aber eine viel spätere Lebensphase JACoBis, in welcher er mit GoEthe schriftlich in Beziehung trat: 1820 wurde er mit der Einrichtung der Irrenanstalt Siegburg bei Düsseldorf betraut. Dadurch wurde $\mathrm{J}_{\mathrm{ACOBI}}$ erst der Psychiatrie zugeführt, in welcher er dann rasch größte Anerkennung erlangte. Die Anstalt Siegburg wurde am 1.Januar 1825 mit JACOBI als Direktor eröffnet. An GoEthe berichtete er in einem sehr ausführlichen Schreiben über die Anstaltspläne und ihre eben erfolgte Gründung (5.Mai 1825) ${ }^{36}$. Auch sandte er ihm den 2. Band seiner «Sammlungen für Heilkunde der Gemütskrankheiten» (Elberfeld 1825), in welchen er die Typuslehre zum allgemeinen Prinzip für die Klassifikation der Geisteskrankheiten erhebt. Man kann wohl im Hinblick auf die durch JACOBI eingeleitete Reform der Irrenpflege sagen: Was für Frankreich Esquirol war, wurde für Deutschland JACoBI: Siegburg wurde zur hohen Schule für die ganze jüngere Generation der damaligen Psychiater Deutschlands.

JAGobi stand wie Nasse als Psychiater auf dem Boden der beobachteten Tatsachen; aber er sah sie in ihrem Zusammenhang als Ausdruck des Wir-

${ }^{35}$ Karl Wigand Maximilian Jacobi, geb. Düsseldorf 10. 4. 1775, gest. Siegburg 18. 5. 1858. Sein in Jena begonnenes Medizinstudium setzte er in Göttingen und in Edinburgh fort, hier um den Brownianismus an der Quelle zu studieren. Nach einigen Jahren der Praxis und weiterer Ausbildung trat er 1805 als Obermedizinalrat in München in den Staatsdienst. 1811-1816 war er Spitalarzt in Salzburg und kehrte dann als Regierungsund Medizinalrat nach Düsseldorf zurück, wo er 1820 mit der Einrichtung der Irrenanstalt Siegburg betraut wurde. Unter seinen Werken sind zu nennen: «Beobachtungen über die Pathologie und Therapie der mit Irresein verbundenen Krankheiten» (1830); «Über Anlegung von Irrenheilanstalten mit ausführlicher Darstellung der Irrenheilanstalt Siegburg» (1838). Sein Hauptwerk: «Die Hauptformen der Seelenstörungen in ihren Beziehungen zur Heilkunde» (1844, Bd. 1, Über die Tobsucht), blieb unvollendet.

${ }^{36}$ F. Th. BratraneK, Goethes naturwissenschaftliche Korrespondenz. Bd. 1, p. 224-230. 
kens einer höheren einigenden Kraft. Dabei blieb er in gewissem Sinne Materialist, d. h. die psychischen Erscheinungen waren ihm nur Äußerungen körperlicher Vorgänge, die psychischen Störungen Symptome körperlicher Krankheiten. Eigentliche Geisteskrankheiten existierten deshalb für ihn nicht. Sitz und Ausgangspunkt der Geistesstörung konnte für ihn irgend ein Organ bilden, das brauchte nicht das «Seelenorgan» zu sein. JACoBI vertrat damit Auffassungen, welche die Neuzeit mehr und mehr bestätigte und welche heute erneut zur Diskussion stehen. JACOBIs Hauptverdienst liegt wohl darin, das genaue Studium des körperlichen Verhaltens während des Verlaufs der Psychosen angeregt und gefördert zu haben. Er beobachtete auf das genaueste die Beziehungen zwischen körperlichen Krankheitszeichen und der Entstehung und dem Verlauf des Irreseins. Der Untersuchung des Pulses und seiner Beziehung zu den Symptomen und Phasen der psychischen Krankheit widmete er große Sorgfalt. In dieser Auffassung traf er sich mit dem ihm befreundeten NASSE, wodurch er mit diesem zum Begründer der somatischen Schule in der Psychiatrie wurde.

Von großer Bedeutung für die Entwicklung der Psychiatrie in Deutschland war auch J. G. LANGERMANN (1768-1832), welcher schon in seiner Inauguraldissertation «De methodo cognoscendi curandique animi morbos stabilienda» (Jena 1797) die psychische Medizin, die er im Sinne StahlS auffaßte, allein auf Beobachtung und Induktion gegründet wissen wollte.

Noch während der Herrschaft der deutschen Naturphilosophie, welche auch die Psychiatrie in Deutschland weitgehend durchsetzte, und in der besonderen Färbung der romantischen Medizin in den Gestalten eines Hahnemann, Mesmer, Eschenmeyer, Passavant und der sonstigen durch die Lehre John Browns erfaßten medizinischen Kreise, auch auf Laien weit übergreifend, eine Epoche psychisch-suggestiver Heilmethoden heraufführte, begann in Deutschland die Reform des öffentlichen Irrenwesens. Und hier ging von LANGermanN ein selbständiger Impuls aus, indem er die Irrenanstalt St. Georgen in Bayreuth neu einrichtete und dadurch den Geisteskranken zu einem menschenwürdigen Dasein verhalf. $1810 \mathrm{kam}$ LANGermann nach Berlin, um die Reorganisation der preußischen Irrenanstalten zu leiten. GoEthe lernte ihn 1812 in Karlsbad kennen. In einem Brief Langermanns aus Berlin vom 18. Juni 1824 an Goethe ${ }^{37}$ beklagte er sich über die Unausrottbarkeit des Charlatanismus und Mystizismus in der Arzneiwissenschaft und über Kollegen, welche seiner eigenen «Bestim-

${ }^{37}$ F. Th. BratraneK, Goethes naturwissenschaftliche Korrespondenz, Bd. 1, p. 244-246. 
mung, Irrenanstalten einzurichten und zu leiten, in welchen auch die Aufregung der geistigen und Willenskräfte versucht werden sollte» mit plumper Hand entgegengetreten seien und sein Werk zunichte machten.

Der Kreis psychiatrisch tätiger Ärzte schließt sich mit Moritz Ennst Adolph Naumann ${ }^{38}$, einem bedeutenden Kliniker, der von 1828 bis zu seinem Tode 1871 in Bonn wirkte und dessen Briefwechsel mit GoETHE von Erich Ebstein ${ }^{30}$ (1922) herausgegeben wurde.

In Goethes Bibliothek steht ein Widmungsexemplar der einzigen in diesem Zusammenhang zu nennenden Schrift Naumanns: «Versuch eines Beweises für die Unsterblichkeit der Seele aus dem physiologischen Standpunkte, zugleich als Einleitung in die Lehre von den sogenannten Geisteskrankheiten, für Ärzte und Gebildete überhaupt» (1830).

Wir haben im vorausgehenden zu zeigen versucht, wie weit GoEthe an der zeitgenössischen wissenschaftlichen Medizin, insbesondere an der Entwicklung der Hirnanatomie, ärztlichen Psychologie, Anthropologie und Psychiatrie teilgenommen hat. Der Versuch einer solchen Darstellung mußte lückenhaft ausfallen und zunächst einmal mehr äußerlich den Spuren folgen, die uns bei solchen Feststellungen leiten müssen. Es mag späteren eingehenderen Studien vorbehalten bleiben, diesen noch wenig erforschten Bereich GoEthescher Beziehungen zur Medizin eingehender zu untersuchen.

Durch diese kleine Studie sollte von einem verhältnismäßig noch wenig erschlossenen Gebiet von GoETHEs wissenschaftlichen Interessen Zeugnis abgelegt werden. Geht doch aus diesen wenigen Beispielen hervor, wie es Goethes unablässiges Bemühen war, zu einer umfassenden Menschenansicht auch von der physischen Seite menschlicher Existenz her zu gelangen und dem Geheimnis der uns auch heute noch so rätselvollen Psychophysik des Menschen durch Vertiefung in die sinnesphysiologischen, hirnanatomischen, psychologischen, psychiatrischen und anthropologischen Probleme, welche seine wissenschaftlichen Zeitgenossen bewegte, eben dem Geheimnis, d. h. dem Urphänomen «Mensch», näher zu kommen.

Den Abschluß bilde ein Wort Goethes, in welchem er, den Drang zur Universalität als notwendig und richtig anerkennend, sagt:

${ }^{38}$ Moritz Ernst Adolph Naumann, geb. Dresden 7. 10.1798, gest. Bonn 19. 10. 1871. Jüngerer Bruder des berühmten Mineralogen KarL Friedrich Naumann.

${ }^{39}$ Erich Ebstein, Aus den Briefen des Klinikers M. E. A. Naumann in Bonn und über seine Beziehungen zu GoEthe. Fortschritte der Medizin, Bd. 40 (1922). 
«Recht gut wissen wir, daß in einzelnen menschlichen Naturen gewöhnlich ein Übergewicht irgend eines Vermögens, einer Fähigkeit sich hervortut und daß daraus Einseitigkeiten der Vorstellungsart notwendig entspringen, indem der Mensch die Welt nur durch sich kennt und also, naiv anmaßlich, die Welt durch ihn und um seinetwillen aufgebaut glaubt. Daher kommt denn, daß er seine Hauptfähigkeiten an die Spitze des Ganzen setzt, und was an ihm das Mindere sich findet, ganz und gar ableugnen und aus seiner eigenen Totalität hinausstoßen möchte. Wer nicht überzeugt ist, daß er alle Manifestationen des menschlichen Wesens, Sinnlichkeit und Vernunft, Einbildungskraft und Verstand zu einer entschiedenen Einheit ausbilden müsse, welche von diesen Eigenschaften auch bei ihm die vorwaltende sei, der wird sich in einer unerfreulichen Beschränkung immerfort abquälen und niemals begreifen, warum er so viele hartnäckige Gegner hat, und warum er sich selbst sogar manchmal als augenblicklicher Gegner aufstößt.» 